\title{
Development and Application of an Industry Foundation Classes-Based Metro Protection Information Model
}

\author{
Yi Zhou, ${ }^{1,2}$ Zhen-Zhong Hu $\mathbb{D}^{1,2}$ and Wei-Zhong Zhang ${ }^{3}$ \\ ${ }^{1}$ Department of Civil Engineering, Tsinghua University, Beijing 100084, China \\ ${ }^{2}$ Graduate School at Shenzhen, Tsinghua University, Shenzhen 518055, China \\ ${ }^{3}$ Guangzhou Mass Transit Engineering Consultant Co., Ltd., Guangzhou 510010, China \\ Correspondence should be addressed to Zhen-Zhong Hu; huzhenzhong@tsinghua.edu.cn
}

Received 2 May 2018; Revised 16 July 2018; Accepted 6 August 2018; Published 26 August 2018

Academic Editor: Mijia Yang

Copyright (C) 2018 Yi Zhou et al. This is an open access article distributed under the Creative Commons Attribution License, which permits unrestricted use, distribution, and reproduction in any medium, provided the original work is properly cited.

\begin{abstract}
Metro protection is the task of evaluating, detecting, and controlling the potential threats presented by external projects to metro structures. To solve the problems encountered in metro protection work, including the isolation of models, difficulties in information extraction, and lack of data processing methods, this study introduced a building information model/modeling (BIM) technology and proposed a metro protection information model (the MPIM) based on the Industry Foundation Classes (IFC) international data standard and its expansion mechanism. According to the information exchange requirements for metro protection, the MPIM defines new customized IFC entities, property sets, and enumeration types to express the models in the metro protection domain. Then the conversion methods of the MPIM and other model formats and the information extraction and application technologies are studied. A metro protection system was developed to implement the MPIM-based model integration and provide assist in preliminary evaluation of external projects via information-rich the MPIM. A trial of the system from Guangzhou Metro's Modiesha Station to Xingangdong Station indicated that the utilization of the MPIM in metro protection workflow could improve working efficiency and save time obviously; management staff claimed that the application of the system saved more than $40 \%$ of the time for project evaluation.
\end{abstract}

\section{Introduction}

Metros are convenient transportation links that positively influence and guide urban planning along the line [1]. Large numbers of new construction projects launched around metro stations are a common phenomenon. Builders of surrounding external projects who conduct relevant civil construction activities need to assess their impact on metro structures, ensure the structural safety of stations and rail transit areas, detect potential safety hazards promptly, and control these hazards. Metro protection is an umbrella term that involves investigations of hidden risks, supervision, daily patrol, external project evaluation, and preparation of various emergency response plans for different situations. Metro protection concerns the interaction between existing metro structures and external project structures and requires comprehensive information, such as that about metro structures, external projects, and surrounding geological information.
Metro protection also determines the influence level of external projects on existing metro structures in conjunction with relevant regulations. Multiparticipants, complex models, and large amounts of information are three majors. However, widespread "information segregation" among models causes many difficulties in the metro protection work.

Building information model/modeling (BIM) technology is a feasible solution to the problem of information isolation in metro protection. This technology aims to solve the long-standing phenomenon of information isolation in the construction industry [2] for the entire life circle of a building. BIM can provide a unified data source and be used among various participants, shared information models, and presentation platforms. In addition, BIMs contain massive information from the construction phase to the O\&M (operation and maintenance) phases. Extracting useful information can provide management personnel with assistance in many aspects, including quick inquiries and aided 
decision-making. Therefore, applying BIM to the working process of metro protection should effectively improve work efficiency.

The concept of BIM was proposed many years ago and has since been successfully applied in many cases, while its adoption in metro protection remains limited. Although Industry Foundation Classes (IFC), as the data format for BIM, has been recognized as the international mainstream in data, the lack of corresponding domain layer entities for IFC in the field of metro protection has resulted in merely partial BIM. The model is homogenized when converted to IFC format, and the information carried is difficult to distinguish and filter and may even be lost directly [3]. Moreover, some parts of the model in metro protection may not be fully compatible with the IFC standard.

Through research and investigation on the process of metro protection work, this study proposes an IFC-based metro protection information model (MPIM), which can effectively integrate different model formats and support data query and decision-making in metro protection work. This research also examines the effect of the MPIM through an indepth application at the Modiesha Station of the Guangzhou Metro. Finally, the further optimization and improvement direction of the MPIM are explored and discussed.

\section{Literature Review}

Metro protection is a subtopic that belongs to the underground safety management. Research in the field of metro safety is remarkably extensive. Relevant scholars have conducted in-depth research and reached significant achievements in data monitoring models, data analysis methods, risk identification algorithms, risk assessment models, and metro operation and maintenance management. For data monitoring, P. J. Bennett et al. [4] installed sensors on the London metro tunnel to develop a PC platform for monitoring the deformation and crack width of the tunnel structure; this work was an early attempt of applying the IoT (Internet of Things) to metro safety management. In terms of security risk identification, Sejnoha [5] proposed an identification tool for risk evacuation in metro structure. The tool classifies all evacuation risks into three categories and uses fault tree analysis (FTA) and event tree analysis (ETA) for quantification analysis. Lu [6] et al. proposed a security risk prediction model utilizing case-based reasoning method, this model stored a large number of existing risk cases as knowledge base and extracted the typical characteristics of typical risks, and then similarity comparison was used to predict potential risks. In risk assessment models, Kyriakidis M. et al. [7] established the definite and quantitative correlation between accident precursors and accident severity by studying a large number of metro safety accidents and then proposed a safety maturity model for describing this correlation. This study has been used successfully in the London Underground to reduce the probability of various types of accidents. Ding [8] proposed a construction risk identification system that is based on plane construction drawings. Through the identification of typical graphic elements in construction drawings, a risk identification knowledge base was established, and a fast matching algorithm based on the knowledge base was adopted to automatically identify potential safety hazards before construction. Wang and Chen [9] combined the fuzzy comprehensive evaluation method and a Bayesian network (BN) to propose a fuzzy-integrated BN model, which quantitatively describes the probability, lose, and controllability of various risks in metro construction projects. Ding and Skibniewski [10] proposed an overall process safety management solution for the construction of metro tunnels, including the system design, safety analysis, risk control, and effect assessment of safety systems. The proposed system was applied to the risk control during construction phase of Wuhan Metro Crossing Tunnels. In the aspect of metro O\&M management, Lyu [11] applied AHP and I-AHP to compare and analyze the flood risk of Guangzhou Metro and assessed the flooding risks of several metro stations, based on existing data. Tian [12] introduced the influence on the metro structure caused by external construction project near the metro and proposed an comprehensive system to monitor and control the settlement, deformation, and stress of the metro structure during the construction phase of the external project. Qiu [13] studied the method of analyzing the safety conditions of metro tunnels under the influence of under-crossing projects.

BIM has developed rapidly in the past decade, and a large amount of research has been conducted on data standards [14-16], information integration [17, 18], and platform development $[19,20]$. Data standards based on IFC [16] have been widely adopted and regarded as the international implementation standard for BIM information exchange. IFC has flexible scalability and can be tailored to specific situations depending on the practical situation. Therefore, IFC is used as a data conversion and storage medium in many studies. Zhang [21] developed a graphic information model (IFCGIM) that is based on the IFC extension. This model uses a new property set defined by the IFC's three existing model entities to store animation properties, which in turn can be used. IFC-GIM realizes virtual construction. Terkaj W [22] studied IFC's semantic expression rules, proposed IFC and Web Ontology Language (OWL, a web-enabled information expression language) conversion methods, and established the ifcOWL model, which uses IWL expressions to describe IFC information to make the IFC model displayed lightly on the web. Afsari K [23] adopted JSON as a medium through which IFC is transmitted to the web. The author expressed the IFC information in JSON format and proposed the concept of the ifcJSON model to implement web-based IFC data transmission. Kang [24] associated IFC with LandXML (a file format exported by the software Civil3D) on the basis of a BIM linkage model and implemented a quick query of information in different format models. Lu [25] believed that BIM model is difficult to be updated in O\&M phase. He used a fuzzy neural network to identify pictures of different components and obtain information such as element texture, material, shape, and size. This method automatically converts pictures to IFC files and can help both BIM researchers and practitioners to develop information-rich BIMs in the O\&M phase. In this way, during the operation and maintenance 
phases of a building, managers can establish a rich BIM information model by capturing pictures. Ding [26] proposed an IFC-based inspection process model, which includes physical, timetable, and quality management models and thus ensures that quality-related information and BIM model information released in real time during construction are smoothly interacted.

In the application aspect, BIM has been applied in all phases of building lifecycle, including design phase, construction phase, and O\&M phase. For example, during the design phase BIM technology can effectively assist in collaborative design [27] and cost analysis [28] on the basis of the data conversion capability of the IFC. During the construction phase, Lu [29] proposed a BIM-based management and reuse method of construction waste. Zhang's $4 \mathrm{D}$ information model is based on BIM technology and realizes virtual construction and construction conflict analysis [30]. In the operation and maintenance phase, studies have been performed on the management and analysis of energy consumption in the construction and maintenance periods using BIM technology [31]. The BIM-based electromechanical equipment intelligent management system BIM-FIM developed by $\mathrm{Hu}$ [18] realized the information sharing during electromechanical equipment installation phase and $\mathrm{O} \& \mathrm{M}$ phase.

BIM also has been applied in the field of metro safety. The Shanghai metro project uses BIM in pipeline collision inspection and installation of large equipment, thereby effectively improving construction quality [32]. Zhang et al. [33] proposed a BIM-based risk identification system and used a BIM model to integrate risk knowledge bases and geometric models. Then the integrated model was used as data source to identify and compare security risks through comparative analyses. The introduction of BIM increases the speed of information extraction and interaction and promotes the sharing of knowledge and communication between customers and domain experts. Marzouk [34] combined BIM technology with a wireless sensor network to achieve temperature monitoring and visualization in metro structures.

On the whole, BIM technology has been successfully applied in metro safety in some aspects. However, after narrowing the scope of metro safety to the field of metro protection, it is discovered that related research applying BIM technology to the work of metro protection remains rare. In fact, BIM with shared and tremendous information is a feasible solution to overcome the information isolation problem in metro protection. Furthermore, extracting vital information from BIM and using data analysis algorithms can be conductive to implement automatic decision-making and raise the informatization level. In short, this aspect deserves further study.

\section{Architecture of the Metro Protection Information Model (the MPIM)}

3.1. Problems and Methods. The process of metro protection work is shown in Figure 1. The metro protection contains two major steps. The first step is the preliminary evaluation. Managers integrate the various types of models and extract vital information that is frequently used in metro protection, such as the horizontal distance between the external project and the metro structure and the depth of the metro, then staff determines the influence level of external projects on metro through a combination of qualitative and quantitative methods. Sometimes the final evaluation conducted by authorities are required when encountered very complex situations. The second step is the daily patrol process. Patrol work will be arranged if the evaluation is passed. Metro protection work considerably differs from ordinary metro O\&M workflow due to the multiple participants involved and lack of unified leadership. Through field surveys and inquiries from managers, the three major problems that currently exist in metro protection work are summarized as below.

\subsubsection{Difficulties in Integration of Different Model Formats.} Managers need to integrate the metro structure model, the surrounding geological model, the external architecture model, and the aerial photography model on the ground. These models are usually created with different software and exported in different formats. IFC is widely supported as the BIM data conversion standard and can effectively integrate different model formats. However, two problems remain. (1) IFC lacks entities suitable for describing the metro protection field. Therefore, most components are described using the IfcBuildingProxy entity when converting to IFC format. Consequently, the information carried by the model becomes difficult to discern and filter and may even be directly lost. (2) Some model formats are still incompatible with IFC standards. For example, the aerial model exported by 3ds Max is generally in FPX format.

3.1.2. Difficulties in Information Extraction. After the model is integrated, managers must examine the relationship between the models and extract valuable information to determine the degree of influence of external projects on the metro structure. The information can be selected and extracted only manually by management personnel. The workload is large, and the efficiency is low. If IFC is used for model integration, the accuracy and speed of information extraction will be limited due to the presence of a large number of homogeneous IfcBuildingProxy entities in IFC.

3.1.3. Difficulties in Data Analysis. The data analysis in metro protection is still mainly relay on manual work and the time cost is quite high. The lack of analysis means is embodied in two aspects: (1) In the preliminary evaluation process, it is necessary to compare the different indexes with the critical conditions after the extraction of essential information. It seems a very tedious process to judge the influence level of external projects. (2) During daily patrol, different patrol frequency should be adopted to different external projects according to their influence levels. However, the project's influence to metro is still a rough indicator. According to previous experience, managers divide the influence into six levels to describe it qualitatively. Therefore, it is difficult 


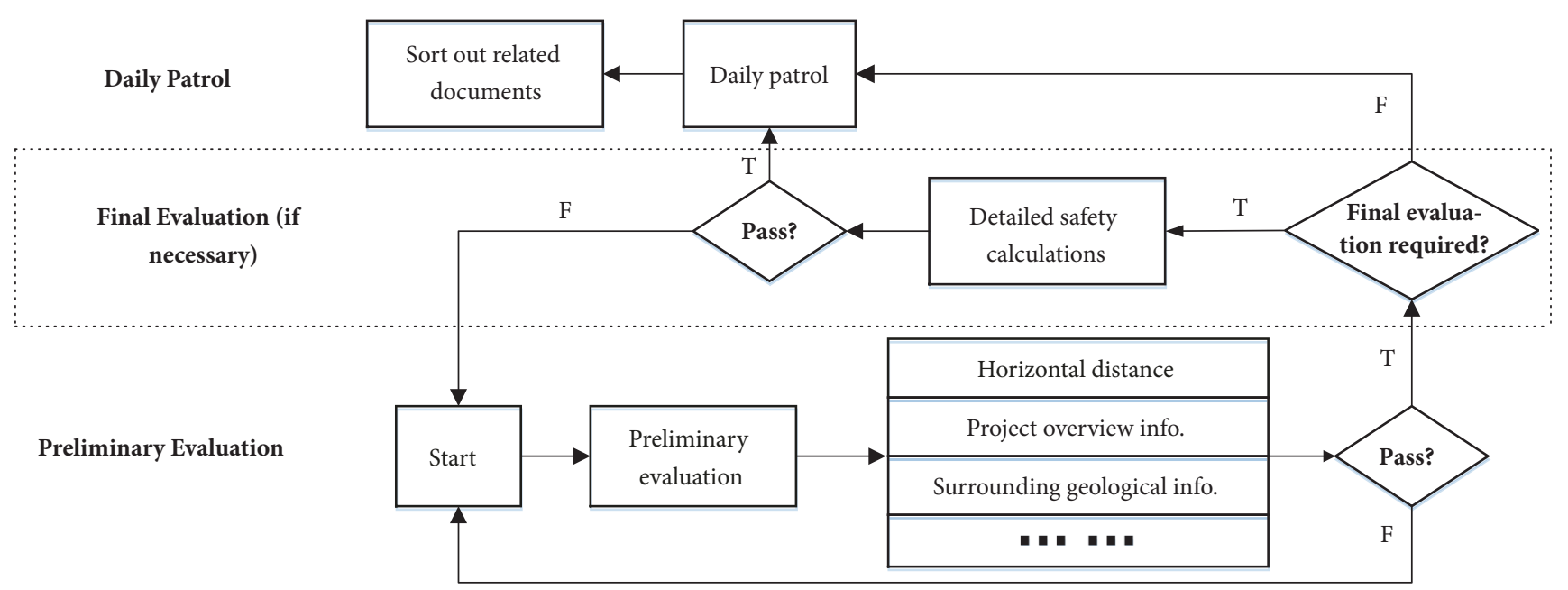

FIGURE 1: A typical workflow of metro protection.

to distinguish the priority of different external projects, especially those with the same influence levels. Managers can only rely on their experience to guide the patrol work. Obviously, a quantitative calculation method is needed to determine the influence level of each external project.

3.1.4. Methods and Algorithms. After full investigation, this research applied a variety of methods and algorithms to solve the aforementioned problems in metro protection. The main algorithms and methods applied are as follows:

(1) Define the metro protection information model. The Industry Foundation Classes (IFC) standard is widely used in representing entities in BIM. The IFC is a kind of object-oriented, rich and neutral schema, and this study proposed a metro protection information model (MPIM) based on IFC. Compared with the original IFC, MPIM defines the specific entities and properties, making the semantic information more accurate and facilitating the subsequent extraction and application of BIM information.

(2) Outline detection algorithm. In the preliminary evaluation process, it is necessary to measure the minimum distance between models. Generally the models contains thousands of triangle meshes and the brute force algorithm by traversal access possesses a very low efficiency. In order to give consideration to both the accuracy and speed of calculation, this study uses the alpha-shape algorithm based on the convex hull as solution. The alpha-shape is an improvement of the convex hull. It adds a parameter $\alpha$ to the convex hull, and when the value of $\alpha$ is suitable, the alpha-shape can describe the irregular contour. This research employed alpha-shape algorithm to extract irregular external outline of large scale models in metro protection and then calculated the minimum clearance between outlines.

(3) Text similarity algorithm based on Levenshtein distance. In this study, in order to realize the quantitative method of the external project's influence level on the metro structure, a large number of text information contained in the patrol records should be processed to extract the related key indicators. Since there are thousands of patrol records to be processed, the text information extraction work should be completed by an automation algorithm. In natural language, there are often multiple similar descriptions of the same concept. In computer these different strings need to be identified and judged to the same meaning. In this study, Levenshtein distance is used to determine the similarity between different strings. Levenshtein distance is the most widely known string metric (also known as edit distance). It operates between two input strings, returning a number equivalent to the number of substitutions and deletions needed in order to transform one input string into another. In this study, if the Levenshtein distance of two strings is small enough, then the similarity is high enough and the two strings are judged to own the same meaning.

(4) External project priority score algorithm based on neural network. As mentioned above, the influence level of external projects is an experiential, rough, and qualitative indicator. The main barrier in quantitative calculation is that the weights of the indicators that affect the influence level are difficult to determine objectively. This study established a neural network to solve this problem. Neural networks are a selflearning model and are particularly suitable for extracting fuzzy relationships or association rules. In learning process, neural network will continuously modify the weight of input layer through backpropagation. In this study, influence indexes are integrated as input vector, and the neural network outputs the probability of each level as a result. Based on the final weights, a quantitative score is calculated to describe the external project's influence. 
MPIM integrates all kinds of information needed in metro protection to form a unified BIM model with complete semantic information, which makes the expression of entities in metro protection clear and explicit. In this study, MPIM will serve as a reliable BIM data source. Many subsequent algorithms will be applied to the process of information extraction and analysis so as to realize the automation in metro protection. The overall solution framework is envisaged and shown in Figure 2.

3.2. Features of the MPIM. To solve these problems in metro protection work, this study proposes an MPIM (Metro Protection Information Model) based on the IFC extension mechanism. The model provides two advantages.

First, the MPIM integrates various formats of models completely. The IFC standard is an international standard that can realize cross-professional, cross-software, and crossplatform data exchange in $\mathrm{A} / \mathrm{E} / \mathrm{C}$ field. IFC also has strong extensibility [8] and can be customized and expanded according to different application domains to improve the accuracy of IFC information expression. Based on the requirements of metro protection work, several IFC shared layer entities are appended in the MPIM utilizing IFC expansion mechanism, thereby ensuring the MPIM with accurate and definite clear property information.

Second, the clear definitions of the metro protection domain in MPIM make it convenient to extract relevant useful information. In the evaluation phase, managers need to obtain various information. These kinds of information include the horizontal distance between different models, the depth of the foundation, the construction method of external project, the support type of foundation pit, the degree of weakness of the surrounding rock, etc. The information provides data support to calculate the influence level of external projects by a series of extraction and analysis methods. In the patrol phase, the external project with the highest priority needs to be identified from the patrol records of various projects. In daily management, all types of documents attached to any project, including monitoring data records, detailed mechanics calculation results, and patrol records, need to be unified and stored in the BIM database for easy querying and sharing. Therefore to make the best of MPIM, the inquiry and data analysis tools and methods are proposed to filter and exploit information carried in MPIM. This scenario is an important manifestation of the realization of BIM value.

3.3. Definition of the MPIM. The IFC models encountered in metro protection work include the metro structure model, the surrounding geological layer model, the external building model, and the ground aerial model. The corresponding four IFC shared layer entities are specifically defined in the MPIM to describe the four aforementioned models. We name these four entities "overall model entity". As to the elements in these models, four other IFC entities are defined and they are named as "model element entity". At the same time, the property set and enumeration type that correspond to each entity are defined. Figure 3 shows the IFC expansion entities and enumerations in the MPIM. A detail that is worth mentioning is that any property value in the property set can be modified under permissions.

In the proposed MPIM, the appended entity IfcMetroBuilding describes the structure of the metro, including the model of the station and rail area. The IfcMetroBuilding entity does not describe the properties of a specific element in the metro structure; rather, it describes the holistic properties of the metro structure. The main properties in its propertyset (Pset_MetroBuilding) include the following: the serviceTime (IfcDuration type), the existence of historical risks (Ifcboolean type), the current risk (IfcPropertyEnumeratedValue type, which can be displayed on the graphics platform with a thumbtack), the safety level (IfcString enumeration type), the average buried depth, the depth of cover soil, and the maximum allowable additional earth stress. The corresponding IfcMetroBuildingEnumType enumeration includes three values, namely, station, section, and default. This enumeration type is used to distinguish whether the metro structure model belongs to a station model or a section tunnel model.

The IfcGeoLayer entity is used to describe the overall properties of the geological layer. The main properties in its propertyset (Pset_GeoLayer) include the grade of the geological surrounding rock (enumeration of integer elements, classified as I, II, III, IV, V, and VI according to the specificationthe default value is 0 ), geological drilling coordinate group (a set of three-dimensional coordinates of the collection type), information under drill points (collection type), the existence of weak underlying layer (IsSoftUnderlying, IfcBoolen type), and underwater depth (IfcMeasuredLength type).

The IfcExternalBuilding entity is defined to describe the overall properties of an external project building. According to the common projects in metro protection, the enumeration IfcExternalBuildingEnumType includes four types of project: medium project, large project, underground pipes, and road/bridge project. The properties are slightly different as the project type varies. When judging influence level to metro, different types of project possess different weights. The external project's propertyset (Pset_ExternalBuilding) contains some of the project's properties that are of highly importance to metro protection managers, such as the project overview (projectSummaries), constructionMethod, baseForm, support type, pit depth, and horizontal distance to metro. For road and bridge projects, whether the foundation of the project is located directly above the metro structure (isOver) needs to be checked.

The IfcGround entity describes the overall properties of the ground aerial model. The entity does not add any predefined properties because it is not essential to describe the overall information of the ground in the metro protection work. But this entity is still necessary for replacing the ambiguous IfcBuildingProxy entity. When managers need to filter the ground model information, the IfcGround can play a role in limiting the traversal scope.

The above are the four overall model entities. For the elements in these models, this study also defined four element entities, including the IfcMetroElement entity, 


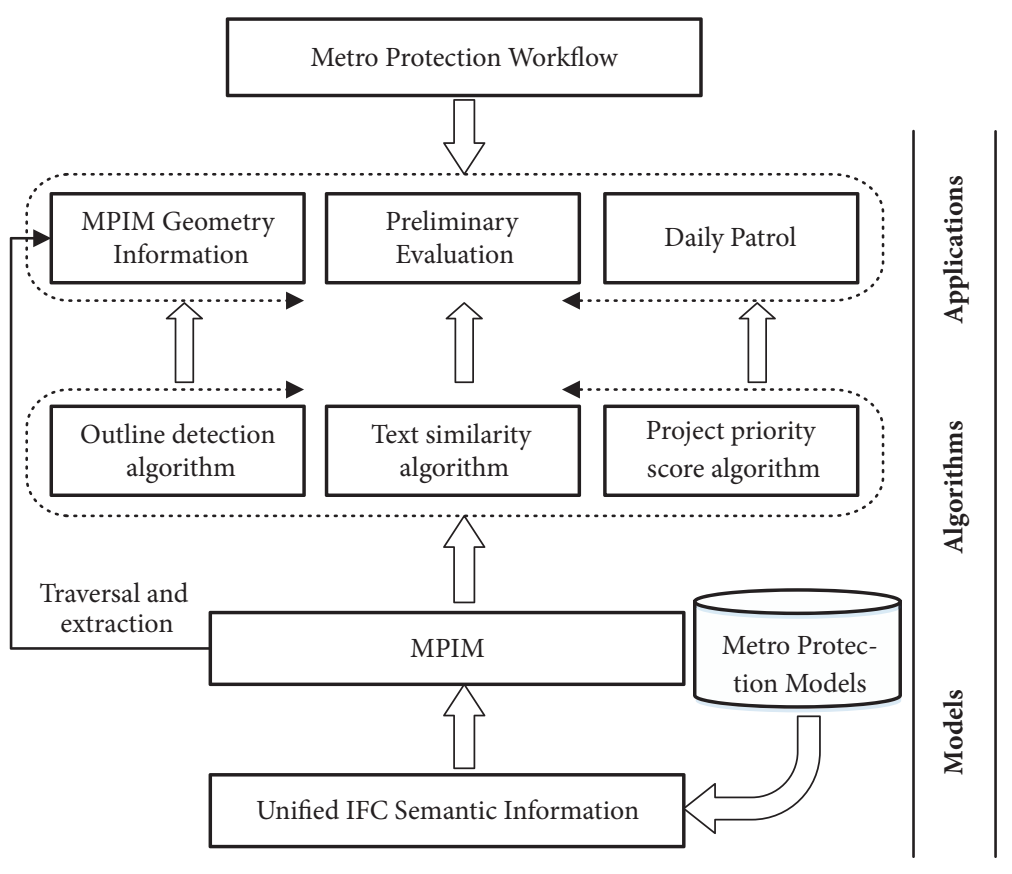

FIGURE 2: Envisaged framework of solution.

IfcGeoLayerElement entity, IfcExternalElement entity, and IfcGroundElement entity.

The IfcMetroElement is used to describe the structural elements of the metro. The properties of its propertyset mainly include some information about metro safety: element type (enumerated type, including platform components, station components, rail zone components, connection layer components, etc.), concrete properties (IfcString type), the last assessment date (IfcDateTime type), the assessment result (IfcString type), the maximum allowable soil stress (IfcPositiveLengthMeasure type, when the value is default, consistent with the current overall model), the current status (IfcPropertyEnumeratedValue type), whether ever repaired (IsRepaired, IfcBoolen type), and service life duration (IfcDuration type). This information helps determine the current health status of the element.

IfcGeoLayerElement is used to describe the properties of geological elements. The predefined enumeration type IfcGeoLayerElementEnumType includes artificial backfill layer, sand layer, clay layer, semiweathered rock layer, rock and soil layer, and rock layer. The propertyset of IfcGeoLayerElement (Pset_GeoLayerElement) mainly includes the soil mechanics properties of the element, such as layer description, bearing load, soil weight, and elastic modulus. When some simple soil stress calculations should be carried out or the degree of weakness of the surrounding rock of the metro needs to be determined, these properties must be extracted.

The IfcExternalElement entity describes the properties of external building elements. The predefined properties of this entity are mainly the material and mechanical information of the element, including the concrete properties (IfcString type), reinforcement information (IfcString type), element volume (IfcPositiveLengthMeasure type), and whether it belongs to the base element (isBaseElement, Nullable Ifcboolean type).

The IfcGroundElement entity describes the properties of the elements in the ground model. In metro protection work, the ground model mainly functions as a reference system to acquire the depth of embedment or excavation of other models. Therefore, for the ground model elements there are two main properties: whether the element belongs to the ground (isGround, Ifcboolean type, which is used to distinguish the element on the ground and the ground itself) and the ground elevation (height, nullable IfcPositiveLengthMeasure type). When an element is not judged to be part of the ground itself, the height property is automatically set to null.

Under normal circumstances, for the above four element entities, if some property information defaults, the information carried by the overall model will be used as a substitute. At the same time, the abovementioned various types of entities will only replace the entities of IfcBuildingElementProxy type. T existing specific IFC entities, such as IfcBeam expressing the beams and IfcSlab expressing the slabs, will not be affected. The extension of the IFC entity makes the description of the model entity accurate and concrete, as shown in Table 1.

Compared with the original IFC, MPIM can extract and traverse the relevant information faster. Metro protection is generally concerned with the overall nature of various models, such as the buried depth of metro structure and the construction method of an external project. According to Table 1, various models are classified elaborately in MPIM. MPIM has specially added various IFC entities for each kind of model, as well as predefined properties holding important information commonly used in metro protection. Then when needed, the information can be found and extracted from 


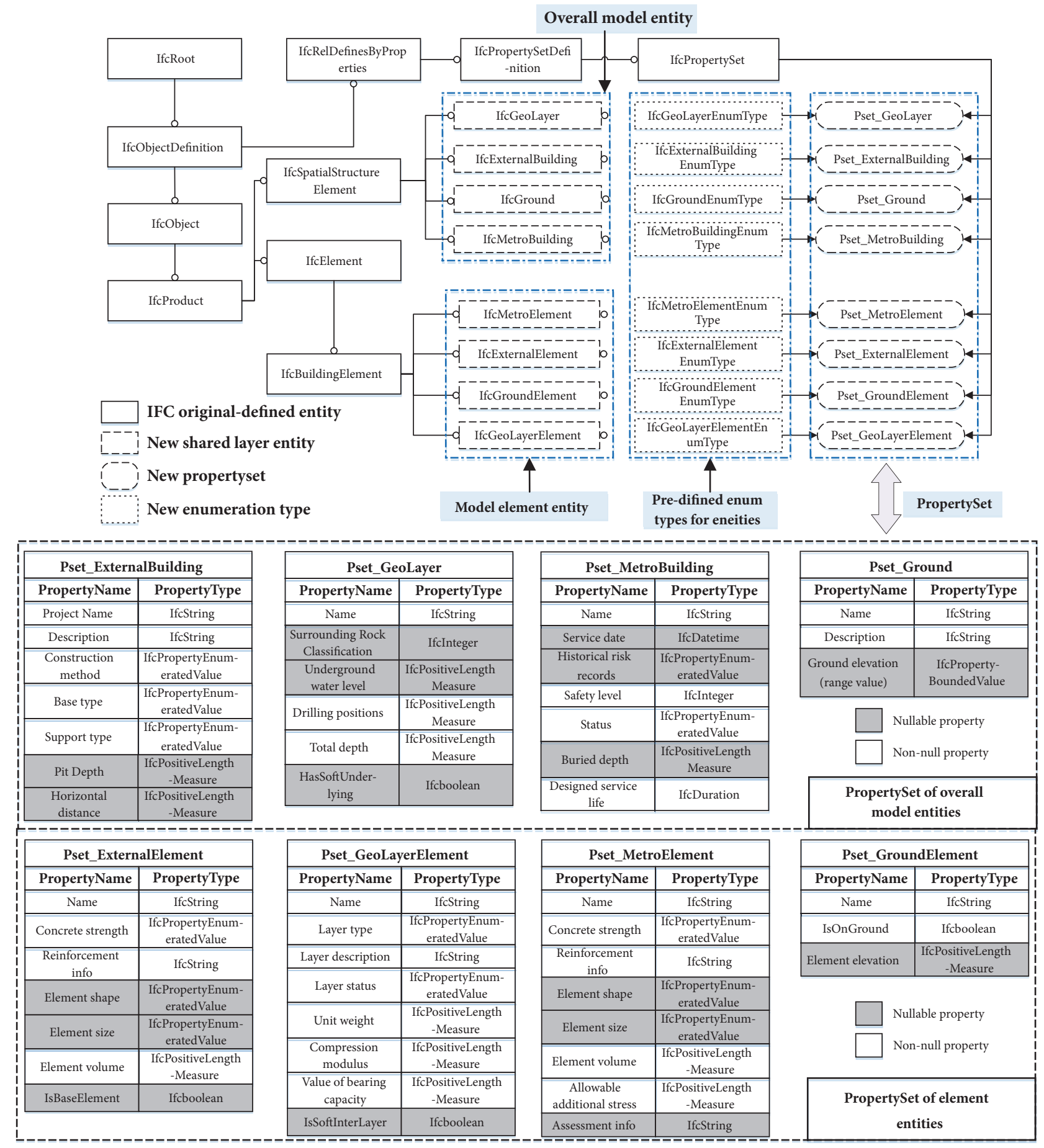

FIGURE 3: IFC expansion of entity, propertyset, and enumeration.

the specific entity quickly. For instance, if the manager wish to obtain the pit depth of an external project, he only needs to traverse IfcExternalBuilding entity and directly extract the value of the property "pit depth". By contrast, in the original IFC, almost all the elements have been stored in the type of IfcBuilding or IfcBuildingElementProxy. There is no detailed entities or predefinition of the related properties. It is difficult to extract any necessary information. In general, we can only use the global traversal method to achieve extraction, which is not only slow, also difficult to guarantee the accuracy of the extracted information. Sometimes it cannot even find the required information and managers have to refer to other relevant files for additional information by manual means. In other words, entities added in MPIM are tags that reduce the traversal scope and the properties provide precise markers of specific information. Figure 4 explains the difference in 
TABLE 1: Comparison between the expanded IFC and the original.

\begin{tabular}{lcc}
\hline Element/Model & Expanded expression & Original expression \\
\hline LayerElement & IfcGeoLayerElement & IfcBuildingElementProxy \\
MetroElement & IfcMetroElement & IfcBuildingElementProxy \\
GroundElement & IfcGroundElement & IfcBuildingElementProxy \\
ExternalElement & IfcExternalElement & IfcBuildingElementProxy \\
ExternalBuilding & IfcExternalBuilding & IfcBuilding \\
GeologicalLayer & IfcGeoLayer & IfcBuilding \\
MetroBuilding & IfcMetroBuilding & IfcBuilding \\
Ground & IfcGround & IfcBuilding \\
\hline
\end{tabular}

information extraction between original IFC and MPIM. Table 2 shows the time cost comparison between MPIM and initial IFC when extracting some common information in metro protection.

3.4. Conversion, Generation, and Storage of the MPIM. Figure 5 shows the MPIM generation and storage process. The MPIM is similar to the origin IFC model except the customized IFC entities it defines. Therefore, for the IFC model, the conversion is relatively simple as long as converting the inappropriate IfcBuildingProxy entity into the corresponding entity in the MPIM. For the non-IFC model, specific plugins can be developed to read those models' information including geometric information, property information, and spatial structure information (general name as semantic information), then the semantic information is written to the corresponding IFC entity in the MPIM and stored in the BIM database. In the specific implementation, this study adopted two model import libraries, namely, XBim and Assimp.

Assimp is a third-party library that supports the reading and importing of many common-format models. Assimp is used in the conversion from non-IFC models to IFC in this study. First, the prewritten plugins read the geometric information and property information in the model. The geometric information mainly refers to the vertex list and index list of the geometric facets of the component. The coordinates of the vertices are transformed into a unified world coordinate system through the transformation matrix, and information such as triangular faces, normal vectors, and bounding boxes of each element is generated and reassembled into a geometric model. For the property information, first it is demanded to judge the category of the geometric model to which the property belongs and then write the property into the corresponding IFC entity according to the category. For example, if the element is determined to be a geological element, then properties are written in the IfcGeoLayerElement entity. If the element is an overall metro element, then all properties are written in the IfcMetroBuilding entity. Some default properties in the propertyset can be generated through geometric information, such as the depth of the metro structure, which can be determined by calculating the average $\mathrm{Z}$ coordinate value of all metro components. At the same time, the corresponding table is created in the BIM database, and the model geometry information and property information can be stored in the corresponding table. Between the geometric model and the properties, the components are linked by an independent GlobalId, and the associations are stored in the corresponding IfcRelSpatialToProperty association table.

In conclusion, the integration of the model is the basis of the entire metro protection work. The MPIM can be used as a reliable BIM data source providing convenience for subsequent information application.

\section{Information Extraction and Application of the MPIM}

The MPIM information is processed and can be applied to many aspects of the metro protection workflow, which mainly includes the following objectives: (1) to achieve safety distance analysis between different macro models based on the information provided in the MPIM; (2) to achieve automatic preliminary evaluation utilizing various information; (3) to achieve a quantitative score method to measure and distinguish the risks of different external projects based on patrol records of metro protection work. The specific implementation is discussed in the following sections.

4.1. Geometric Information Extraction and Application. For the surrounding projects submitted for evaluation and approval, the first step in preliminary evaluation is to estimate the minimum distance between the project and the metro structure. Taking into account the fact that the practical project is mostly approximately perpendicular to the ground plane, this study projects the model onto the $\mathrm{Z}=0$ plane, then calculates the distance between the projections, and then in turn calculates the distance between the three-dimensional models. The alpha-shape algorithm is used to generate the outer contour of projection. To reduce the amount of calculations and errors, two main measures are taken:

(1) The elements located inside the model obviously cannot contain the two closest points. Therefore, before the projection, the internal elements are eliminated by a judging algorithm.

(2) The influence of external building on the metro structure is exerted through the foundation; thus only the elements belonging to the building's foundation are considered in the calculation. In other words, cantilever elements are not taken into consideration.

The algorithm is divided into three steps. The first step is to screen the vertices of the model elements that meet the conditions and project them to the $\mathrm{Z}=0$ plane. The second step is to generate the alpha-shape hull on the basis of the plane vertex set and to calculate the minimum distance between the hulls and finds the nearest two points. The third step is to find the nearest two points in the space model by finding the nearest point on the plane. The second step to obtain the outline of the model is the core of the algorithm. Its pseudocode is shown in Algorithm 1.

4.2. Property Information Extraction and Application. In the preliminary examination of metro protection, several key 


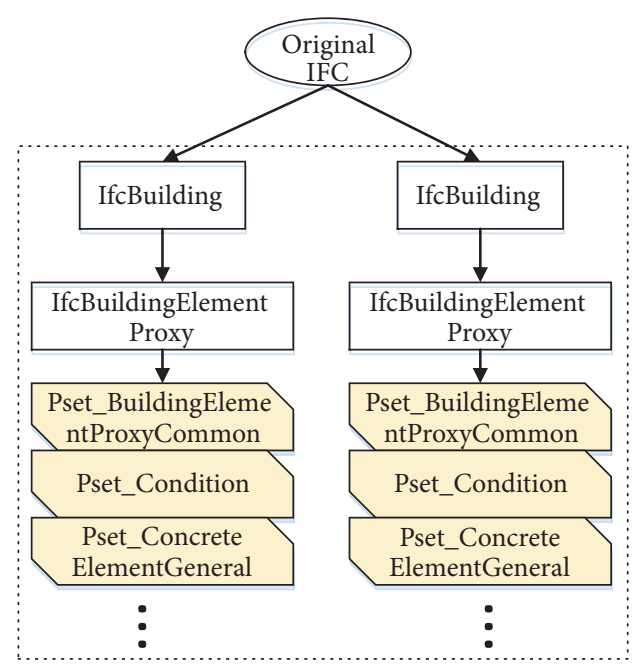

Traversal Scope in Original IFC

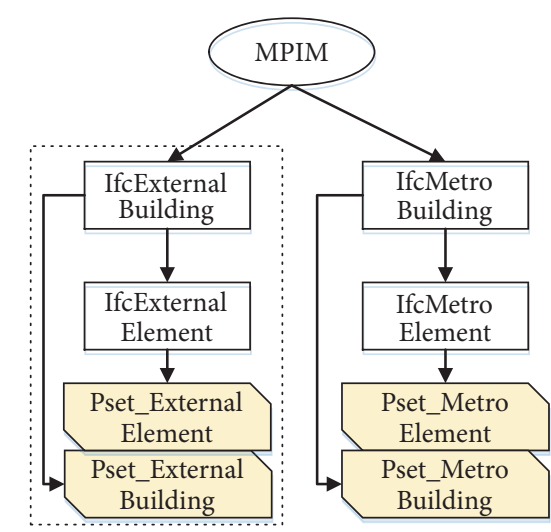

Traversal Scope in MPIM

1. MPIM significantly reduces the traversal scope.

2. Original IFC usually owns many redundant propertyset while lacks specific properties required in Metro Protection

FIgURE 4: The difference in information extraction between original IFC and MPIM.

TABLE 2: Information extraction time cost of MPIM and original IFC.

\begin{tabular}{lcccc}
\hline Type & $\begin{array}{c}\text { Construction Method } \\
\text { (external project) }\end{array}$ & $\begin{array}{c}\text { Construction Method } \\
\text { (metro project) }\end{array}$ & Pit depth & $\begin{array}{c}\text { Surrounding Rock } \\
\text { Level }\end{array}$ \\
\hline Original IFC & $73 \mathrm{~ms}$ & $65 \mathrm{~ms}$ & $91 \mathrm{~ms}$ & $83 \mathrm{~ms}$ \\
MPIM & $9 \mathrm{~ms}$ & $7 \mathrm{~ms}$ & $12 \mathrm{~ms}$ & $42 \mathrm{~ms}$ \\
\hline
\end{tabular}

indicators need to be checked to determine the degree of influence of external projects on the metro structure. A specification [35] pointed out that the influence of external project's operations on the metro structure is divided into six levels: superfine level, level 1, level 2, level 3, level 4, and level 4-, as shown in Table 3.

The influence level is judged by two direct indicators: the proximity degree and the influence zone. The proximity degree is mainly determined by the distance between the metro structure and the external building $L$ and the depth of the metro structure $H$. The influence zone is affected by the excavation depth of the external building, and the surrounding rock grade, external building support type, etc. are also considered factors. The MPIM described by the expanded IFC contains accurate information. By comprehensive utilizing this information, managers can extract the abovementioned various types of values to assist the preliminary evaluation (Table 4).

\subsection{Attachment Information Extraction and Application. In} metro protection work, a large number of patrol records are uploaded to the metro supervision server every day. The staffs need to review each of these records to find out the most urgent and prior projects that should be handled first. However, the traditional manual inspection method not only takes a long time but also lacks efficiency because it lacks a quantitative calculation method for the priority judgment of different projects. Even though the regulation [35] gives the method to judge the influence of the external project, the result is still relatively rough and the manager has to relay on experience in a great extent. Metro protection patrol records are textual information, but its characteristics are different from those of ordinary text and characters. (1) The patrol records are usually written according to a predefined template with a relatively fixed format and writing order. (2) The patrol records are highly professional texts; some professional words have high frequency of occurrence and carry large amounts of information. Through these special words, the useful information that needs to be extracted in the patrol records is usually clear.

With the use of the information, the corresponding method was proposed to achieve the risk score for the metro protection patrol records. The steps of this process are as follows:

(1) Define the keywords to confirm the information needed to be extracted. The keywords are determined through consulting relevant managers and then a keyword database is established.

(2) Word segmentation is first performed. In this study, an open-source segmentation scheme [36] was used to predefine common nouns in a number of patrol records, and then all the records are segmented and nonmeaningful stoppages are eliminated.

(3) The keywords have been identified in step (1), while each keyword in the record often has different expressions in patrol records. "The minimum horizontal distance" and "the minimum horizontal projection 


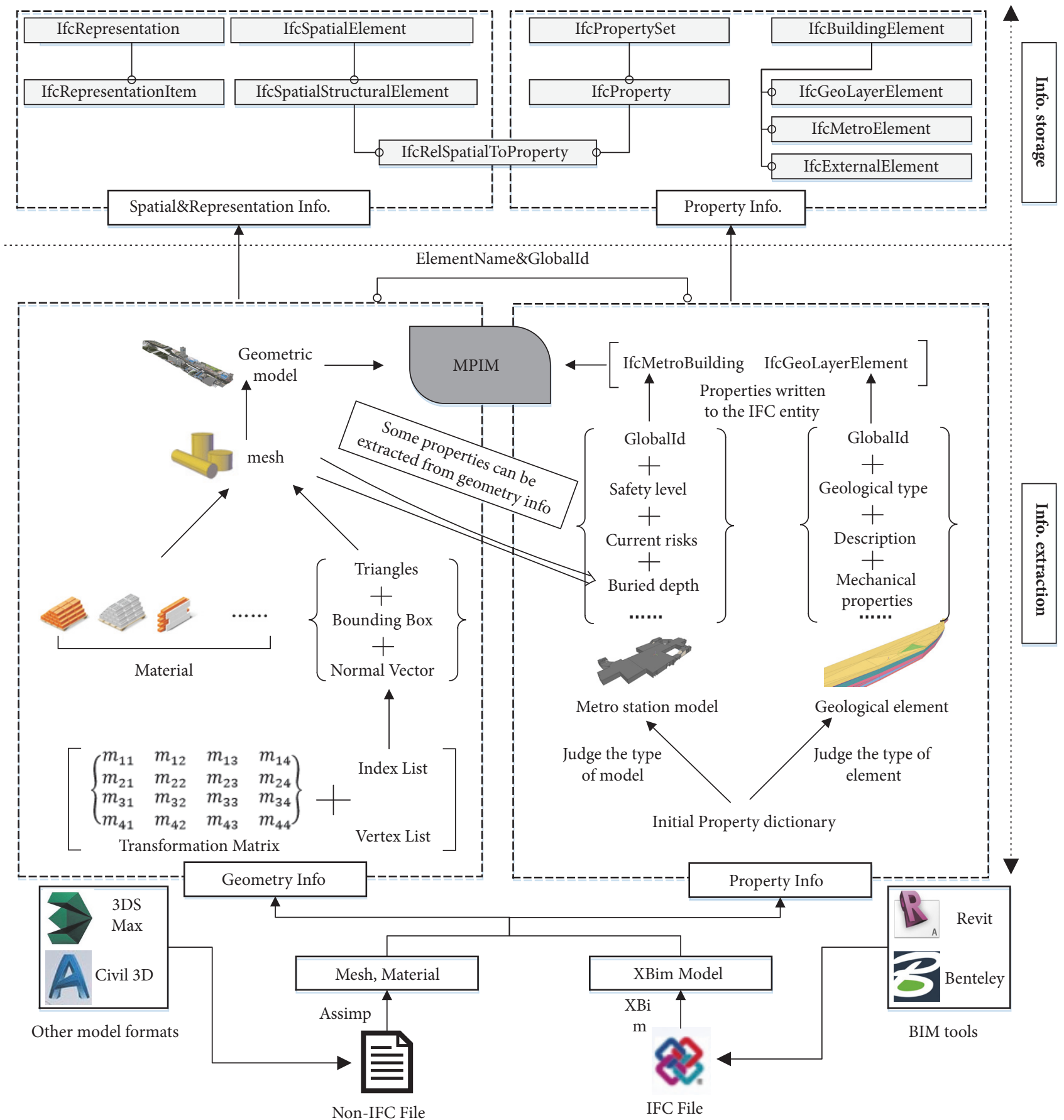

FIGURE 5: The generation and storage of the MPIM.

TABLE 3: Division of external project influence level.

\begin{tabular}{lcccc}
\hline Influence & & & Proximity & \\
& Very close & close & Partly close & Not close \\
\hline Strong influence (A) & Superfine level & Superfine level & Level 1 & Level 2 \\
Significant influence (B) & Superfine level & Level 1 & Level 2 & Level 3 \\
General influence (C) & Level 1 & Level 2 & Level 3 & Level 4 \\
Lower influence (D) & Level 2 & Level 3 & Level 4 & Below level 4 \\
\hline
\end{tabular}

Note. If there exists soft underlying layer or soft interlayer in the surrounding geologic model of the metro structure, the influence level will be raised by one level. 


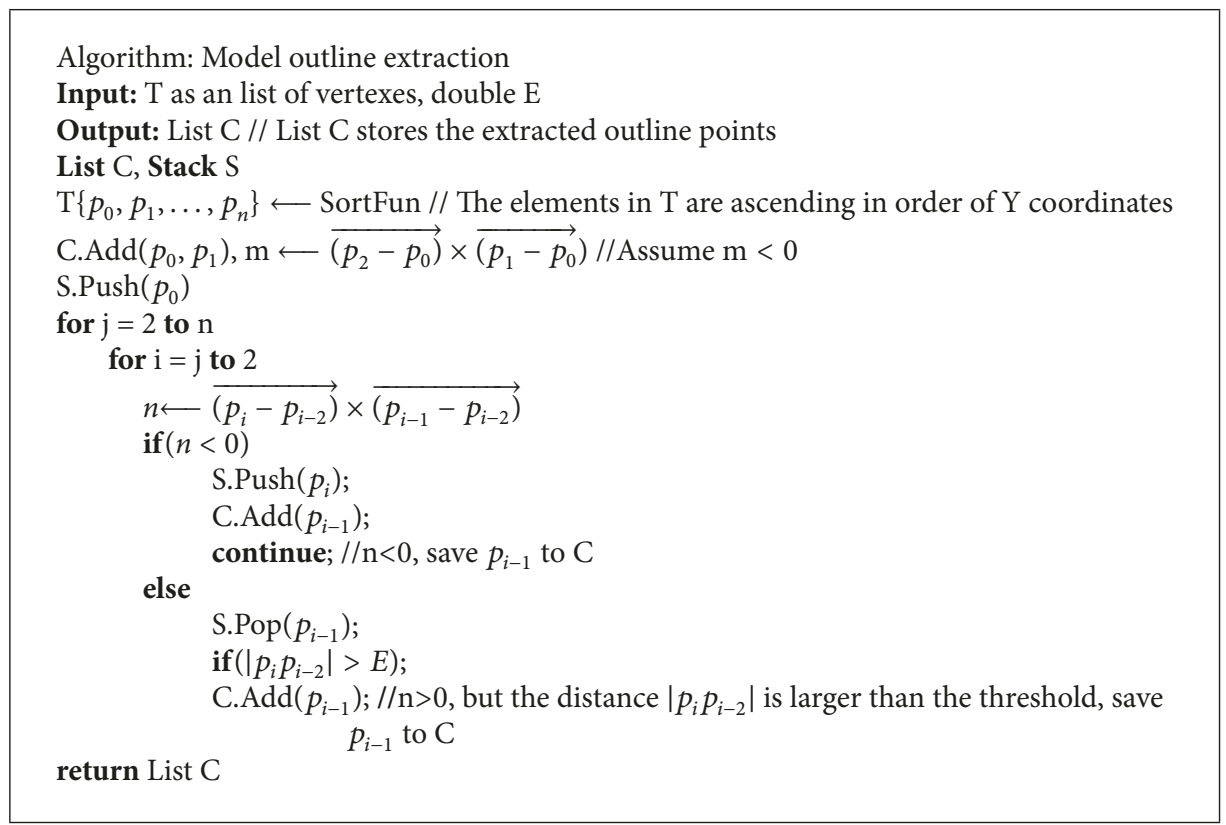

Algorithm 1: Algorithm to extract model outline.

TABLE 4: Extracting major information from the MPIM.

\begin{tabular}{|c|c|c|}
\hline Major factors & Extraction method & IFC type uesd \\
\hline $\begin{array}{l}\text { The horizontal distance } L \text { between the } \\
\text { external project and the metro } \\
\text { structure }\end{array}$ & Utilize Algorithm 1 to calculate L. & All IFC types \\
\hline Buried depth $H$ of metro structure & $\begin{array}{l}\text { Traverse the IfcGround and IfcMetroBuilding model, } \\
\text { filtering the elements with the largest } \mathrm{Z} \text { coordinate } \\
\text { difference and } \mathrm{H}=|\mathrm{Z}| \text {. }\end{array}$ & $\begin{array}{l}\text { IfcGround } \\
\text { IfcMetroBuilding }\end{array}$ \\
\hline Pit depth $h$ of external project & $\begin{array}{l}\text { Traverse the IfcGround and IfcExternalBuilding, filtering } \\
\text { the elements with the largest } \mathrm{Z} \text { coordinate difference and } \\
\text { get } \mathrm{H}=|\mathrm{Z}|\end{array}$ & $\begin{array}{l}\text { IfcGround } \\
\text { IfcExternalElement }\end{array}$ \\
\hline Grade of surrounding rock & $\begin{array}{l}\text { Judge from the property IsSoftInterLayer and } \\
\text { IsSoftUnderlying }\end{array}$ & $\begin{array}{l}\text { IfcGeoLayer } \\
\text { IfcGeoLayerElement }\end{array}$ \\
\hline Support type & $\begin{array}{l}\text { Judge from the property Support Type in } \\
\text { IfcExternalBuilding entity }\end{array}$ & IfcExternalBuilding \\
\hline
\end{tabular}

distance" represent the same meaning. Therefore, to correctly extract effective information from different expressions, string matching algorithm is adopted. This study uses a Levenshtein distance-based decision algorithm. Levenshtein distance can determine exactly how many editing changes need to be operated to make two strings exactly the same. The editing here includes three operations: insert, delete, and replace. If the strings are more similar, then the calculated Levenshtein distance is smaller. Suppose we need to compare the two string arrays $a=\left(a_{1}, a_{2}, \ldots, a_{n}\right.$, $)$ and $b=\left(b_{1}, b_{2}, \ldots, b_{m},\right)$; the string arrays represent an expression of keyword, while the string element in the array represents a single word. The Levenshtein distance calculation process is shown as

$$
\begin{aligned}
& d_{[i, j]} \\
& = \begin{cases}0 & i=0 \text { or } j=0 \\
\min \left(d_{[i-1, j]}+1, d_{[i, j-1]}+1, d_{[i-1, j-1]}\right) & a_{i}=b_{j} \\
\min \left(d_{[i-1, j]}+1, d_{[i, j-1]}+1, d_{[i-1, j-1]}+1\right) & a_{i} \neq b_{j}\end{cases}
\end{aligned}
$$

Two string similarities are defined based on the Levenshtein distance $d_{[i, j]}$, as shown in

$$
S=1-\frac{d_{[i, j]}}{\max (\text { a.length, b.length })}
$$

For example, it only takes one delete operation to make two strings "the minimum horizontal distance" 


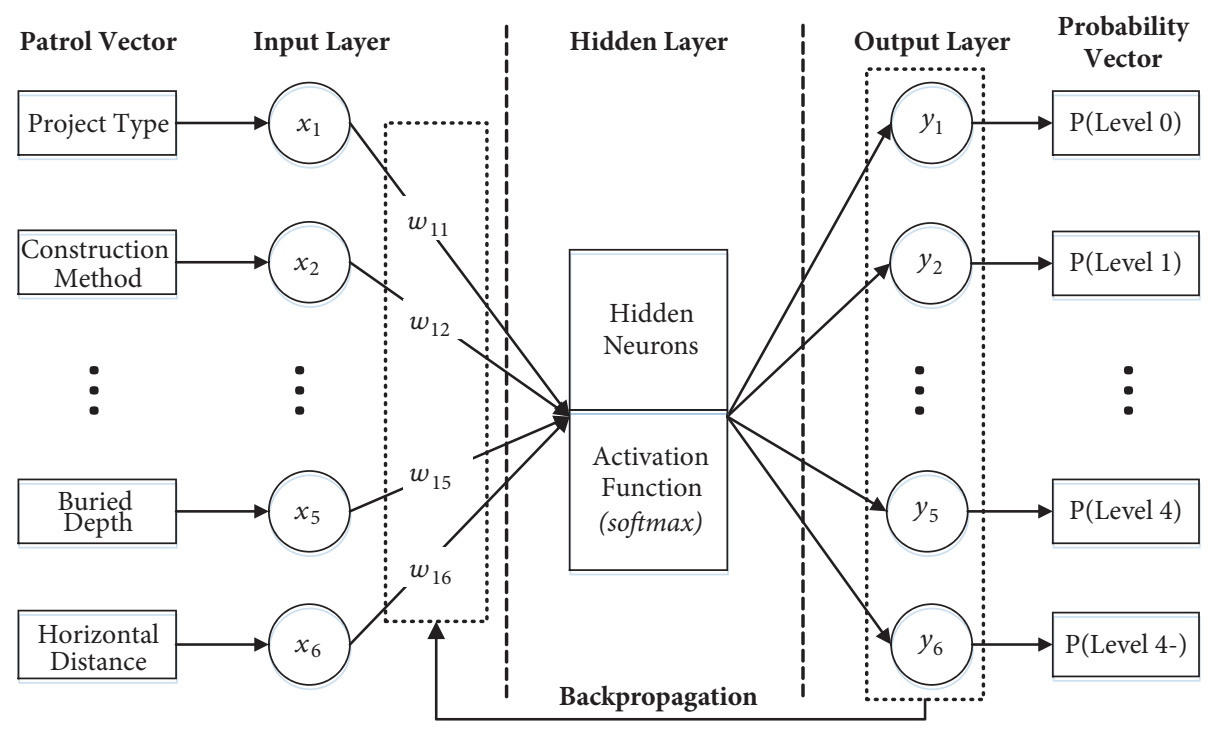

FIGURE 6: Working process of BP neural network.

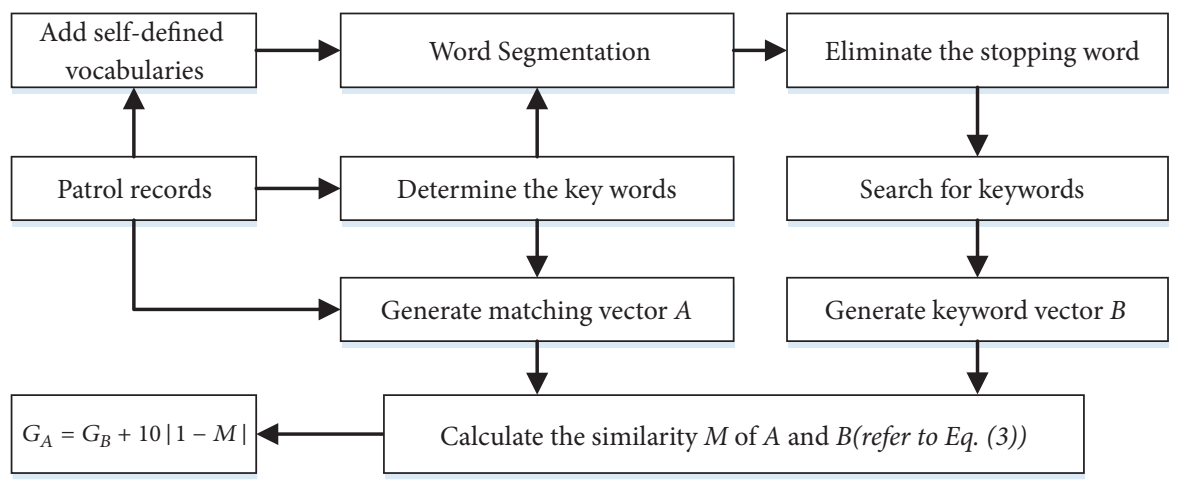

FiguRE 7: Flow chart of priority assessment for patrol records.

and "the minimum horizontal projection distance" exactly the same so $d_{[i, j]},=1$ and $S=0.8$.

To increase the fault tolerance rate, several common keyword expressions are added in advance to keyword database. The target string will calculate the similarity $S$ with all these expressions when searching keywords, and the boundary values $S_{\text {max }}$ and $S_{\text {min }}$ are collected as index. When $S_{\max } \geqslant 85 \%$ and $S_{\min } \geqslant$ $50 \%$, the two strings can be determined to have the same meaning.

(4) Keywords are similar to pointers. After finding the keyword in the patrol record, we can search the information we need in keyword's context. Then, a patrol vector $A\left(a_{1}, a_{2}, \ldots, a_{n}\right)$ is generated. Each component of vector $A$ represents the key information of corresponding keyword. On the basis of the related standards, several reference vectors $B\left(b_{1}, b_{2}, \ldots, b_{n}\right)$ are generated in advance. Table 5 shows a patrol vector $A$ extracted from one single record and the reference vector $B$ with the same influence level used for matching and comparison. Some of the components in patrol vector are string format, we need to transform them to digital format. We give different transform weights $W_{\text {tran }}$ to different enumeration values under the same keyword. For example, the project type, as a keyword, has three available enumeration values: medium project $\left(W_{\text {tran }}=1.0\right)$, large project $\left(W_{\text {tran }}=\right.$ $1.2)$, and road/bridge project $\left(W_{\text {tran }}=1.3\right)$; different string values would transform to the corresponding $W_{\text {tran }}$.

(5) When the components of patrol vectors are transformed into digital types, another question came up: how should the weight of each component of the vector be determined? This study chooses BP neural network to avoid subjectivity in weight's setting. The input is the patrol vector, and the output results are the probability vector that each component indicates the probability of corresponding influence level. Trained with a large number of existing patrol records, the $\mathrm{BP}$ neural network will learn and adjust the weights of the input layer. After 1000 training in trial, the accuracy of the general network can exceed $96 \%$. At this time, 
TABLE 5: Patrol records information extracted by keywords.

\begin{tabular}{lccccccc}
\hline keyword & Project type & $\begin{array}{c}\text { Influence } \\
\text { level }\end{array}$ & $\begin{array}{c}\text { Construction } \\
\text { method }\end{array}$ & Soft under-lying & Pit depth & $\begin{array}{c}\text { Buried depth } \\
\text { distance }\end{array}$ \\
\hline Vector A & Large & Level 2 & Open cut & False & $8.2 \mathrm{~m}$ & $16.4 \mathrm{~m}$ & $15.1 \mathrm{~m}$ \\
Vector B & medium & Level 2 & Open cut & False & $5 \mathrm{~m}$ & $15 \mathrm{~m}$ & $15 \mathrm{~m}$ \\
\hline
\end{tabular}

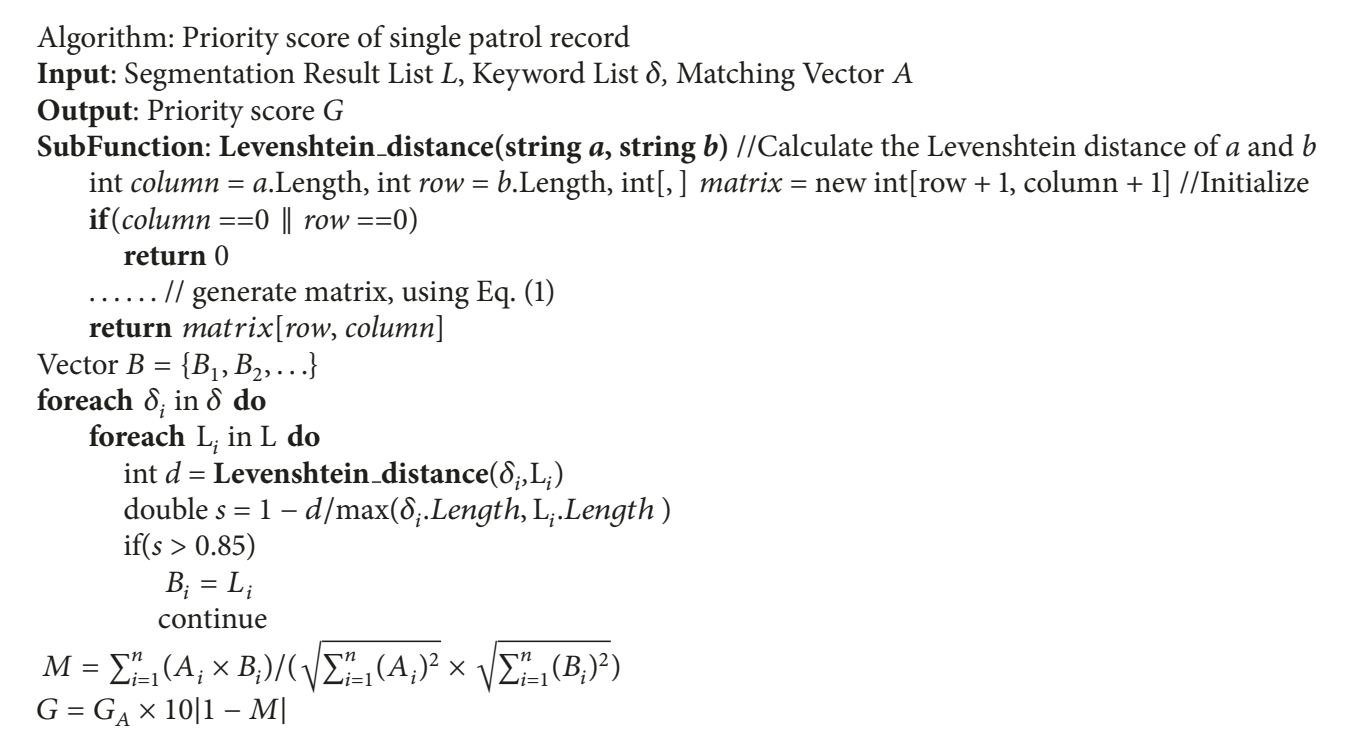

Algorithm 2: Pseudocode of priority assessment for one single patrol record.

the weights $W$ of the input layer in the BP network are extracted as weights of the patrol vectors used in following score calculation. The working process of BP neural network is shown in Figure 6.

(6) The similarity between vector $A$ and corresponding $B$ is calculated as a measure of the urgency and priority of one patrol record. In the set of reference vectors, vector $B$ that possesses the same level of influence as vector $A$ is selected, and the cosine similarity is used to measure the similarity $M$ between $A$ and $B$, as shown in (3). On the basis of $B$ 's base risk score $G_{B}$, $A$ 's risk score $G_{A}$ is calculated as shown in

$$
\begin{aligned}
M & =\frac{\sum_{i=1}^{n}\left(W_{i} A_{i} \times W_{i} B_{i}\right)}{\left(\sqrt{\sum_{i=1}^{n}\left(W_{i} A_{i}\right)^{2}} \times \sqrt{\sum_{i=1}^{n}\left(W_{i} B_{i}\right)^{2}}\right)} \\
G_{A} & =G_{B}+10|1-M|
\end{aligned}
$$

The entire process diagram is shown in Figure 7. The pseudocode for the scoring algorithm is shown in Algorithm 2.

In this algorithm, suppose the word segmentation result of a patrol record contains $m$ words, the total number of keywords is $k$, the current keyword string length is $n$, and the length average value of each time the string is compared with the keyword is also approximately $n$. Then, the overall time complexity is $\mathrm{O}\left(\mathrm{kmn}^{2}\right)$. As a predefined keyword, the number $k$ will not change. The methods of reducing the value of $m$ and $n$ are the main means to reduce the calculation time. This study uses the following methods to optimize the efficiency of the algorithm:

(1) Decrease the average length $n$ of the matching string. In the Levenshtein distance calculation, if the completely identical substrings in two strings are eliminated in advance, then this elimination will not affect the final calculation result, but it can effectively reduce the matrix size generated by the calculation process. The effect is especially noticeable for two strings with high similarity.

(2) Reduce the number of strings (words) $m$ that need to be matched. The patrol records have a fixed format. The contents of the records are classified according to different topics, such as the project profile, the relationship with the metro, and the pit depth. Different keywords can be queried in different blocks. When matching keywords, managers only need to examine the content that is close to the keyword. For example, when matching the keyword "construction method", managers only need to search for the "project profile" part of the patrol record to reduce the number of invalid matches.

Table 6 shows the optimization effect after using these two optimization methods in the processing of the keyword "horizontal distance" in one patrol record. 
TABLE 6: Optimization results of one patrol record.

\begin{tabular}{lcccc}
\hline & Number of words & Average length of words & No. of words to be matched & Total match time \\
\hline before & 34 & 2.62 & 34 & $141 \mathrm{~ms}$ \\
after & 34 & 2.60 & 14 & $68 \mathrm{~ms}$ \\
\hline
\end{tabular}

\section{Case Study}

5.1. System Development. A BIM-based metro protection system is developed based on the MPIM, previous described algorithms, and an existing BIM-FIM [10] system. Adopting the $\mathrm{C} / \mathrm{S}$ (client/server) logical framework, the overall system architecture is shown in Figure 8. In general, the system is divided into five layers, namely, the server layer, model layer, platform layer, application layer, and interface layer. The server layer provides the user with model files, interfaces for uploading and importing various types of attachments, and algorithms for reading and converting model data. This layer is also responsible for managing the rights of all users and organizations. The model layer divides all model information into geometric information, spatial structure information, property information, and volume information, and reassembles them. The geometric and spatial structure information generate a spatial element tree, and the property and mass information are used. A newly defined IFC entity is then generated to be described. The IFC entities and geometry components are linked using GlobalId to form a complete MPIM. The platform layer is a three-dimensional display tool of models based on the rendering and the optimization techniques. The application layer is based on the unified BIMFIM platform and develops related functions, extracts and utilizes effective information in the MPIM, and assists the metro protection managers to implement automation and informatization, such as the computer-aided evaluation and quantitative score.

5.2. Practical Case Application Process. The presented system was applied to the metro protection work at the Modiesha-Xingangdong section of the Guangzhou Metro. Modiesha is a station on Line 8 of the Guangzhou Metro. It is located in Haizhu District and is connected to South China Expressway and suffers heavy traffic. Light industry companies and residential areas gather near the station, and new projects around the station are carried out frequently. The system uses the model of a residential building as an example of an external project. The whole process of system application is as follows.

5.2.1. Establishment of the MPIM. Revit was adopted to establish the geological layer model and the metro structure model, while ground aerial models were mostly processed by $3 \mathrm{ds}$ Max, and the modeling of external projects varies according to the project type. The building project was dominantly modeled by Revit, industrial plants by Tekla, and the gallery by Bentley's PowerCivil. Figure 9 shows the process of model integration process.

In this project, the models included the metro model and the external project model. The metro model included the
Modiesha station model, the interval geological model, the interval orbit model, and the ground aerial model. The aerial model was established in 3ds Max, and other models were established by Revit. Two file model formats existed: Revitexported IFC files and 3ds Max-generated FBX files. With the use of the model processing method of this study, the FBX file was imported with Assimp, and the XBim file was imported into the IFC. After the different models were imported, their properties were written into the corresponding IFC entities. The MPIM was generated after the property information was associated with the geometric information, and then integrated in the BIM-FIM platform to achieve a threedimensional display.

5.2.2. Information Extraction and Application of the Entities. After the MPIM was established, the information extraction algorithms and tools developed in the system sped up the information screening, filtering and querying, to achieve aided-evaluation in metro protection. Figure 10 shows the five-step process of the extraction and application of information to obtain recommended external building influence levels.

(i) For information that could be read directly from the properties of relevant IFC entities, such as the construction method of the external building, the type of the foundation pit support, and so on, the system traversed the corresponding IFC entities and extracted the existing properties as needed information. Step 1 and step 2 indicate the aforementioned process.

(ii) For information that could not be obtained directly, according to the relevant algorithm, the system used existing information and provided algorithms for calculation and generation. For example, through the geometric information of the metro structure and the external building, the system extracted outline of project and metro structure, so as to calculate the minimum horizontal distance, step 3.1 shows that a satisfactory outline was obtained in the proper threshold, and the minimum distance was calculated as $27.7 \mathrm{~m}$. By reading and comparing the bearing capacity properties and type properties of each geological layer in the geological model, the system could determine whether there exists soft underlying or soft interlayer around the metro structure. Step 3.2 shows this process.

(iii) In the end, combined with comprehensive information, the recommended influence level of external projects on the metro structure was provided to assist the evaluation of metro protection. Steps 4 and 5 reveal the process. 


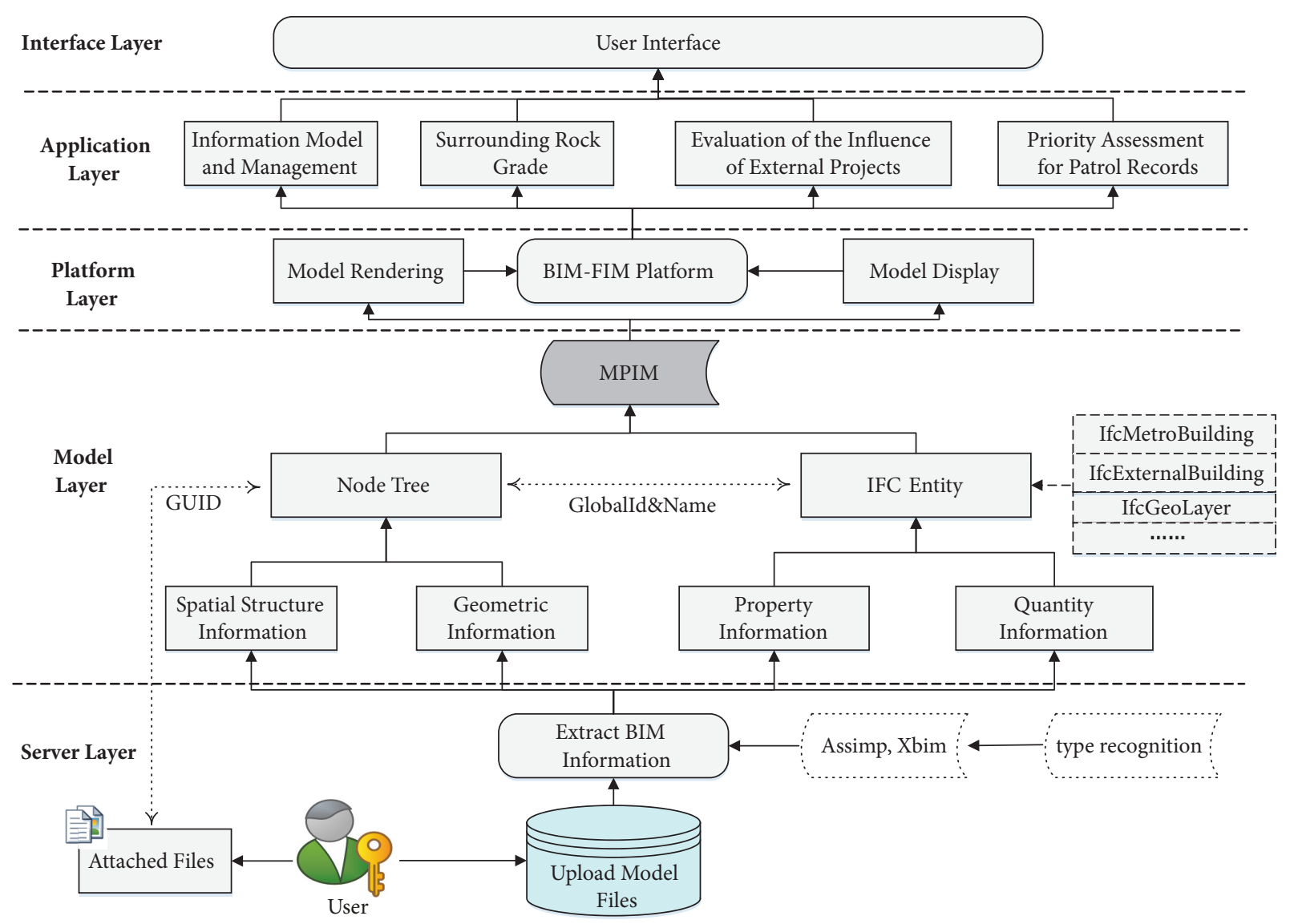

FIgURE 8: The BIM-based metro protection system architecture based on the MPIM and the BIM-FIM.

5.2.3. Information Extraction and Application of Attachment. Although the MPIM takes rich information, a considerable part of the information cannot be carried in the form of properties, and it needs to be associated with the BIM model through attachments, such as patrol records, calculation books, various contracts, certification documents, and monitoring data. The use of this information is also an important function of the system. In this example, some patrol records from December 2016 to December 2017 were extracted. On the basis of the algorithm proposed in Section 4.2, seven keywords were identified: "project type", "minimum horizontal distance", "pit depth" (of external project), "buried depth"(of metro station), "soft underlying", "construction method", and "influence level". Each keyword has an enumeration as spare parts for comparison. As a result, each patrol record will extract these seven pieces of information to form a vector $\mathrm{A}\left(a_{1}, a_{2}, a_{3}, a_{4}, a_{5}, a_{6}, a_{7}\right)$. In accordance with the specifications, several matching vectors $B\left(b_{1}, b_{2}, b_{3}, b_{4}, b_{5}, b_{6}, b_{7}\right)$ are generated according to the differences in levels of influence, construction methods, and project types. These vectors will be used for references and matching; a comparison is then performed. Based on the benchmark priority score that $B$ owns, the system calculated the calculated similarity between $A$ and $B$ and the priority of the project in the $A$ record will be calculated. Table 7 shows the seven keywords' information of two patrol records. Vector A is extracted from patrol record sheet while vector B is generated as matching vector. Record B's influence level is level 1 , so it owns a basis priority score of $80^{\prime}$. In the calculation of similarity, the ratio of horizontal distance to pit depth (name $r_{1}$ ) and the ratio of horizontal distance to buried depth (name $r_{2}$ ) are the most vital index. Horizontal distance still needs to be considered, we calculate the ratio of the two records' horizontal distance as another index (name $r_{3}$ ). Then scale vector A based on B. For the same components in vector, in order to reduce its impact on computation, they will be set to 0 . The similarity grade $\mathrm{M}=$ 0.936 is calculated via (3).

\section{Discussion}

Compared with the ordinary IFC model, the MPIM provides more accurate descriptions of relevant information in the field of metro protection and can effectively overcome the long-standing problem of information segregation in metro protection. Information can be shared between different models. According to the MPIM, the BIM-based metro protection system was developed based on the existing BIMFIM of the research group. The system can integrate different models in the form of the MPIM under the same platform and provide a variety of functions and algorithms for automatic extraction and generation of various types of required 


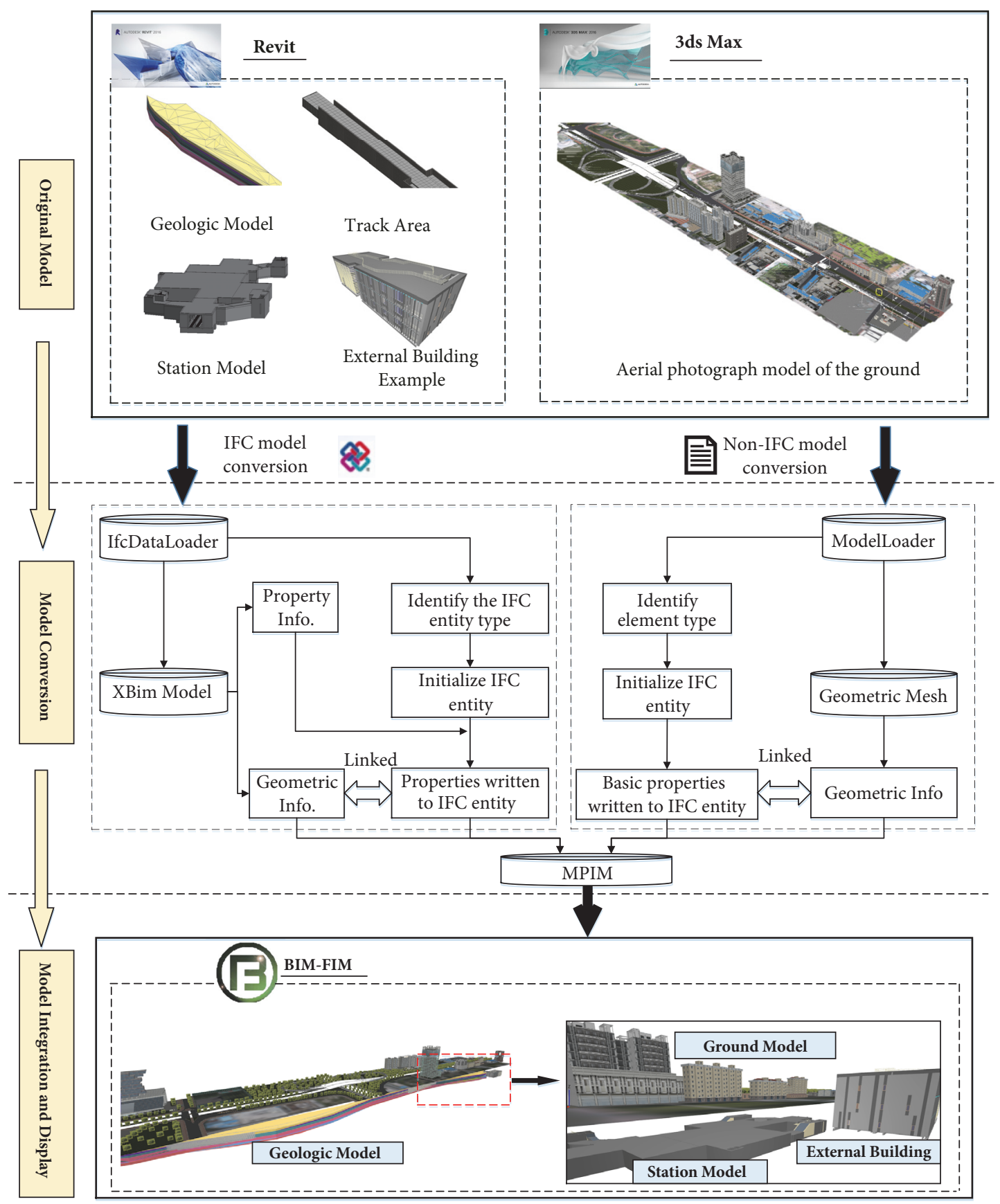

Figure 9: Generation and display of the MPIM.

information in the metro protection field. For example, the distance between the metro structure and the external project is automatically calculated, and the surrounding rock grades are determined based on the properties of the geological layer components. Finally, the influence level of the external project on the metro structure is combined with various types of effective information to identify the more dangerous items in the massive number of patrol records. A more complete coverage of the metro protection workflow is thus achieved.

The system was applied to the metro protection work in the Modiesha-Xingangdong section of the Guangzhou
Metro. The system covered the entire protection work process, including model integration, model view, preliminary review, and patrol record risk identification. Information integration, visualization, and information sharing are the three most valuable benefits that the MPIM brought to metro protection. According to the metro protection staff, all models were integrated into the same platform, and the MPIM contained rich and accurate information. The sharing of information formats sped up the screening of important information. At the same time, various functions provided by the system realized computer-aided activities 
TABLE 7: The patrol vector generated from records.

\begin{tabular}{|c|c|c|c|c|c|c|c|}
\hline & Project type & Influence level & Construction method & Soft underlying & $r_{1}$ & $r_{2}$ & $r_{3}$ \\
\hline Vector A & 1.2 & 1 & 0 & 0 & 0.62 & 0.92 & 1.01 \\
\hline Vector B & 1.0 & 1 & 0 & 0 & 1.0 & 1.0 & 1.0 \\
\hline \multicolumn{4}{|c|}{$L=$ Horizontal distance, $B d=$ Buried depth, $P d=$ Pit depth. } & \multicolumn{4}{|c|}{$r_{1}=L / P d, r_{2}=L / B d, r_{3}=L_{A} / L_{B}$} \\
\hline
\end{tabular}
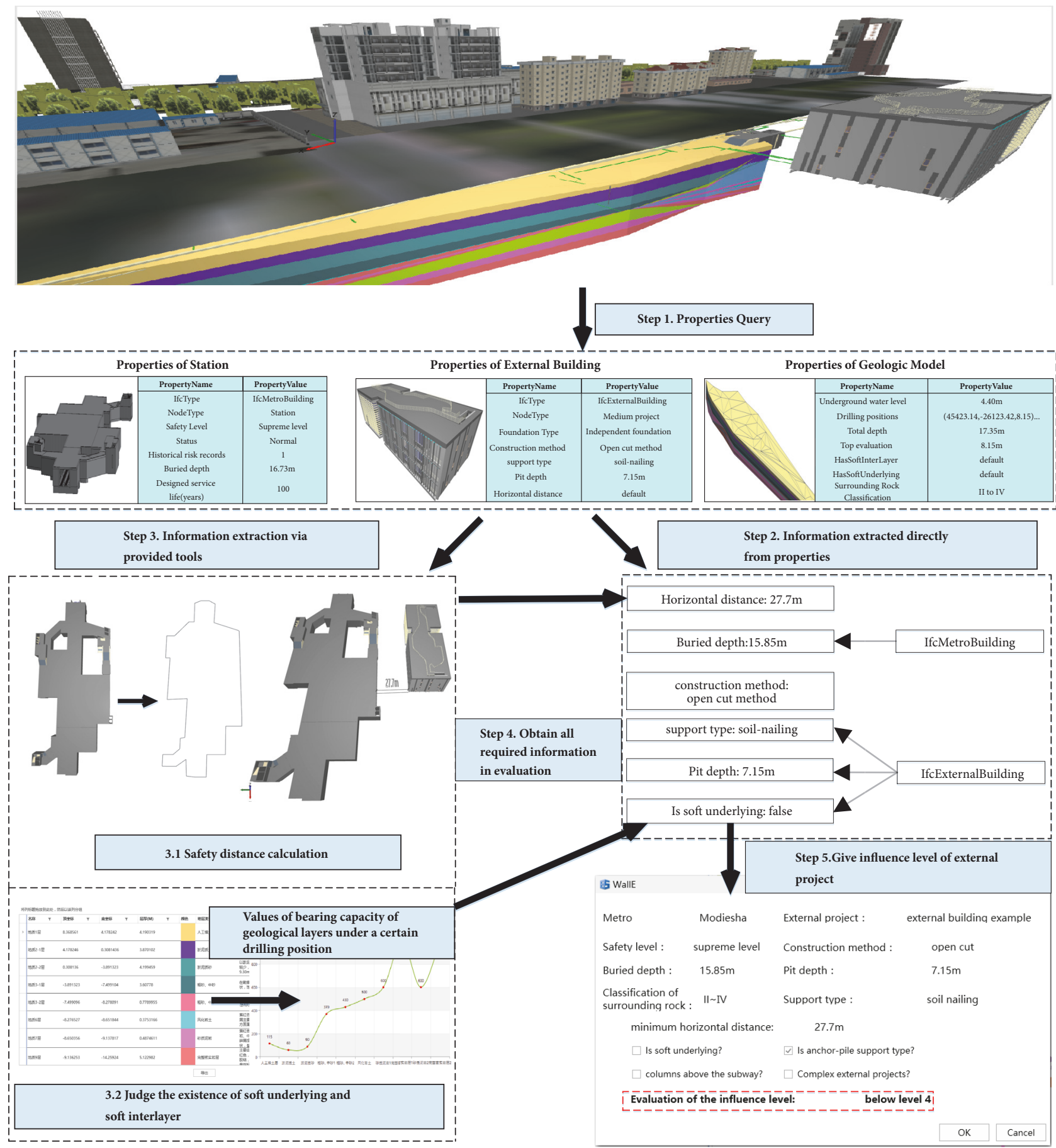

FIGURE 10: Information extraction and application of the MPIM. 
for various tasks in metro protection. The efficiency of the metro protection work was obviously improved. Managers claimed that even considering the manual verification time, the average evaluation time for an external project was reduced by about $40 \%$. As for the daily patrol work, according to the algorithm provided by the system, identifying the more dangerous external projects in the patrol records reached the accuracy rate of $90 \%$. In the implementation of the algorithm, the conservative calculation method was adopted. In the misjudged items, all the risk degree of the external project was overestimated. The results of the algorithm were partial to the safety.

However, some problems and barriers still existed in the application of the MPIM and the metro protection system. The first problem is that, in the process of model conversion, inconsistencies in the world coordinate system occurred frequently. Although the local engineering projects in Guangzhou were required to use the Guangzhou urban construction coordinate system as the benchmark, in metro protection work, the aerial photography model was widely used, and its coordinate system was generally based on the WGS-84 geospatial coordinate system. The conversion process that took place between the WGS-84 coordinate system and the urban construction coordinate system in Guangzhou was complicated. More crucially, aerial photography models often lacked sufficient reference points, which led to inaccurate coordinates of aerial models even if they understand the corresponding coordinate transformation methods. The solution to this problem was to establish sound modeling and aerial specifications and to strengthen communication and management in subsequent applications.

The second problem was the strong coupling issue between various models. Many types of models were involved in the metro protection project. The focus was on the relationship between different types of models. If a model was missing, the function of the entire system was affected significantly. For example, if no ground model was given, unless the property was explicitly written out, the buried depth of the metro structure could not be calculated because of the lack of a reference surface, which greatly affected the subsequent judgment with regard to the influence level of external projects and even directly results in erroneous results. Determining a way to minimize the coupling between models to the utmost extent and improve the robustness of the system is a concern in follow-up work and development.

The lack of a related mobile platform was the third barrier. Only development on the PC side was taken into consideration due to the limited development time of this system. Daily inspection work was a vital part of the metro protection. Inspection personnel needed to fill in the patrol records on site and upload them to the designated database, thus the demand on the mobile terminal was urgent. Currently, the system could only manually store the daily patrol records in the BIM database through management personnel. Thus, development of a supporting mobile platform to achieve automatic uploading and storage of patrol records was necessary.

\section{Conclusion and Future Work}

This study proposes an IFC-based MPIM to address the problem of information isolation in metro protection. Then a MPIM-based metro protection system was developed and after application trial in the Guangzhou Metro, the following conclusions are drawn:

(1) The MPIM information model is based on the IFC extension mechanism. The geometric information and property information are closely connected through GlobalId and form semantic information. Compared with the common IFC model, the new IFC entity for metro protection in the MPIM ensures that the MPIM describes the metro protection field more accurately, prevents the loss of information, and has better identification ability and querying speed for important information. Through research of the general model and the MPIM transformation mechanism, the integration of different format models is realized, thereby effectively overcoming the information isolation between models and enabling the sharing of all information.

(2) The metro protection system developed based on the MPIM can cover the main workflow of metro protection. Not only can the system realize the integration of all information, but it can also be applied to the screening and extraction of effective information in the MPIM. Various algorithms and tools assist metro protection managers in the evaluation of external projects and the priority analysis of patrol records. After trials, management officials stated that the system could shorten the time for reviewing external projects by an average of $40 \%$.

The development of mobile platforms that support the current system will be the focus of future work. In view of the space breadth of the patrol work, introducing GIS technology into metro protection work is of great significance. In addition, the next step in future work is to ensure that the system itself has the ability to identify hidden dangers by utilizing neural networks and other deep learning models with the use of a large number of photos and texts for training.

\section{Data Availability}

The data used to support the findings of this study are available from the corresponding author upon request.

\section{Conflicts of Interest}

The authors declare that they have no conflicts of interest.

\section{Acknowledgments}

This research was supported by the National Natural Science Foundation of China (no. 51778336 and no. 51478249) and the Tsinghua University-Glodon Joint Research Centre for Building Information Model (RCBIM). The authors would 
like to acknowledge the Metro Protection Department of the Guangzhou Metro for providing the application case and data support.

\section{References}

[1] W. Yong-bing and C. Hai-hua, "Research on sustainable development of urban metro traffic in China," China Population, Resources and Environment, vol. 26, pp. 141-144, 2016.

[2] P. J. Bennett, Y. Kobayashi, K. Soga, and P. Wright, "Wireless sensor network for monitoring transport tunnels," Proceedings of the Institution of Civil Engineers: Geotechnical Engineering, vol. 163, no. 3, pp. 147-156, 2010.

[3] X.-Y. Zhang and Z.-Z. Hu, "Research on model conversion approach towards structural finite element analysis," Gongcheng Lixue/Engineering Mechanics, vol. 34, no. 6, pp. 120-127, 2017.

[4] W. N. MacPherson, M. Silva-Lopez, J. S. Barton et al., "Tunnel monitoring using multicore fibre displacement sensor," Measurement Science and Technology, vol. 17, no. 5, pp. 1180-1185, 2006.

[5] J. ejnoha, D. Jarušková, O. Špačková et al., "Risk quantification for tunnel excavation process. Proc. World Acad. Sci," in Proceedings of the World Academy of Science, Engineering and Technology, vol. 58, pp. 101-109, 2009.

[6] Y. Lu, Q. Li, and W. Xiao, "Case-based reasoning for automated safety risk analysis on subway operation: Case representation and retrieval," Safety Science, vol. 57, pp. 75-81, 2013.

[7] M. Kyriakidis, R. Hirsch, and A. Majumdar, "Metro railway safety: an analysis of accident precursors," Safety Science, vol. 50, no. 7, pp. 1535-1548, 2012.

[8] L. Y. Ding, H. L. Yu, H. Li, C. Zhou, X. G. Wu, and M. H. Yu, "Safety risk identification system for metro construction on the basis of construction drawings," Automation in Construction, vol. 27, pp. 120-137, 2012.

[9] Z. Z. Wang and C. Chen, "Fuzzy comprehensive Bayesian network-based safety risk assessment for metro construction projects," Tunnelling and Underground Space Technology, vol. 70, pp. 330-342, 2017.

[10] L. Ding, L. Zhang, X. Wu, M. J. Skibniewski, and Y. Qunzhou, "Safety management in tunnel construction: Case study of Wuhan metro construction in China," Safety Science, vol. 62, pp. 8-15, 2014.

[11] H.-M. Lyu, W.-J. Sun, S.-L. Shen, and A. Arulrajah, "Flood risk assessment in metro systems of mega-cities using a GIS-based modeling approach," Science of the Total Environment, vol. 626, pp. 1012-1025, 2018.

[12] T. Jingliang, Research and Fulfillment for Constructing New Buildings in Metro Protection Area, Tianjin University, Tianjin, China, 2014.

[13] Q. dongyi, The deformation monitoring and analysis for existing metro tunnel under the influence of traversing engineering, Beijing Jiaotong University, Beijing, China, 2012.

[14] C. Eastman, P. Teicholz, R. Sacks, and K. Liston, BIM Handbook: A Guide to Building Information Modeling for Owners, Managers, Designers, Engineers And Contractors, Wiley, New York, NY, USA, 2008.

[15] NBIMS-US, "Building Information Model Standard-United States Version 3," BuildingSMART Alliance, BIM Committee Report, 2015.

[16] R. Vanlande, C. Nicolle, and C. Cruz, "IFC and building lifecycle management," Automation in Construction, vol. 18, no. 1, pp. 7078, 2008.
[17] Y.-N. Park, Y.-S. Lee, J.-J. Kim, and T. S. Lee, “The structure and knowledge flow of building information modeling based on patent citation network analysis," Automation in Construction, vol. 87, pp. 215-224, 2018.

[18] Z.-Z. Hu, P.-L. Tian, S.-W. Li, and J.-P. Zhang, "BIM-based integrated delivery technologies for intelligent MEP management in the operation and maintenance phase," Advances in Engineering Software, vol. 115, pp. 1-16, 2018.

[19] J. Choi, J. Shin, M. Kim, and I. Kim, "Development of openBIMbased energy analysis software to improve the interoperability of energy performance assessment," Automation in Construction, vol. 72, pp. 52-64, 2016.

[20] Z. Liu, Y. Li, X. Lu, and H. Zhang, "BIM-based integrated information framework for architectural and structural design model," Tongji Daxue Xuebao/Journal of Tongji University, vol. 38, no. 7, pp. 948-953, 2010.

[21] J. Zhang, F. Yu, D. Li, and Z. Hu, "Development and implementation of an industry foundation classes-based graphic information model for virtual construction," Computer-Aided Civil and Infrastructure Engineering, vol. 29, no. 1, pp. 60-74, 2014.

[22] W. Terkaj and A. Šojić, "Ontology-based representation of IFC EXPRESS rules: An enhancement of the ifcOWL ontology," Automation in Construction, vol. 57, pp. 188-201, 2015.

[23] K. Afsari, C. M. Eastman, and D. Castro-Lacouture, "JavaScript Object Notation (JSON) data serialization for IFC schema in web-based BIM data exchange," Automation in Construction, vol. 77, pp. 24-51, 2017.

[24] T. W. Kang, "Object composite query method using IFC and LandXML based on BIM linkage model," Automation in Construction, vol. 76, pp. 14-23, 2017.

[25] Q. Lu, S. Lee, and L. Chen, "Image-driven fuzzy-based system to construct as-is IFC BIM objects," Automation in Construction, vol. 92, pp. 68-87, 2018.

[26] L. Ding, K. Li, Y. Zhou, and P. E. D. Love, "An IFC-inspection process model for infrastructure projects: Enabling real-time quality monitoring and control," Automation in Construction, vol. 84, pp. 96-110, 2017.

[27] S. Barg, F. Flager, and M. Fischer, "An analytical method to estimate the total installed cost of structural steel building frames during early design," Journal of Building Engineering, vol. 15, pp. 41-50, 2018.

[28] W. Lu, C. Webster, K. Chen, X. Zhang, and X. Chen, "Computational Building Information Modelling for construction waste management: Moving from rhetoric to reality," Renewable \& Sustainable Energy Reviews, vol. 68, pp. 587-595, 2017.

[29] Z. Hu and J. Zhang, "BIM- and 4D-based integrated solution of analysis and management for conflicts and structural safety problems during construction: 2. Development and site trials," Automation in Construction, vol. 20, no. 2, pp. 155-166, 2011.

[30] P. Pishdad-Bozorgi, X. Gao, C. Eastman, and A. P. Self, "Planning and developing facility management-enabled building information model (FM-enabled BIM)," Automation in Construction, vol. 87, pp. 22-38, 2018.

[31] R. Khan, Z. Aziz, and V. Ahmed, "Building integrated agriculture information modelling (BIAIM): An integrated approach towards urban agriculture," Sustainable Cities and Society, vol. 37, pp. 594-607, 2018.

[32] Q. Qian and P. Lin, "Safety risk management of underground engineering in China: Progress, challenges and strategies," Journal of Rock Mechanics and Geotechnical Engineering, vol. 8, no. 4, pp. 423-442, 2016. 
[33] S. Zhang, J. Teizer, J.-K. Lee, C. M. Eastman, and M. Venugopal, "Building Information Modeling (BIM) and Safety: automatic safety checking of construction models and schedules," Automation in Construction, vol. 29, pp. 183-195, 2013.

[34] M. Marzouk and A. Abdelaty, "Monitoring thermal comfort in subways using building information modeling," Energy and Buildings, vol. 84, pp. 252-257, 2014.

[35] Housing and urban-rural development department of Guang Dong, Technical Code for Protection of Existing Structures of Urban Rail Transit, 2009.

[36] "Jieba Chinese Word Segmentation Solution[EB/OL]," https:// github.com/fxsjy/jieba. 


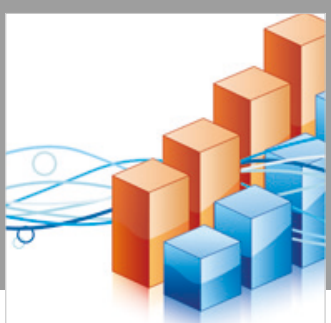

Advances in

Operations Research

\section{-n-m}
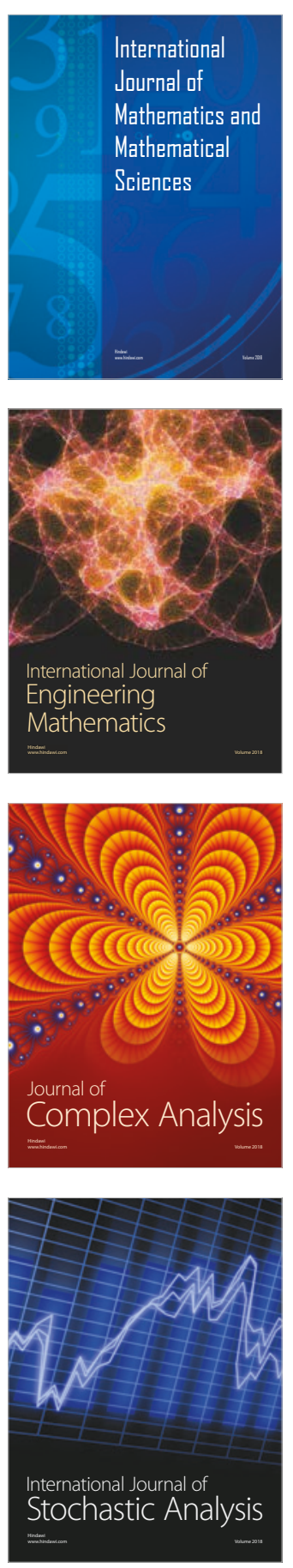
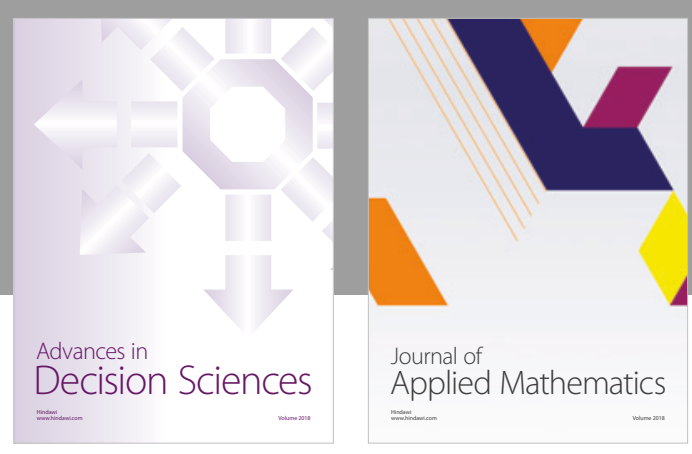

Journal of

Applied Mathematics
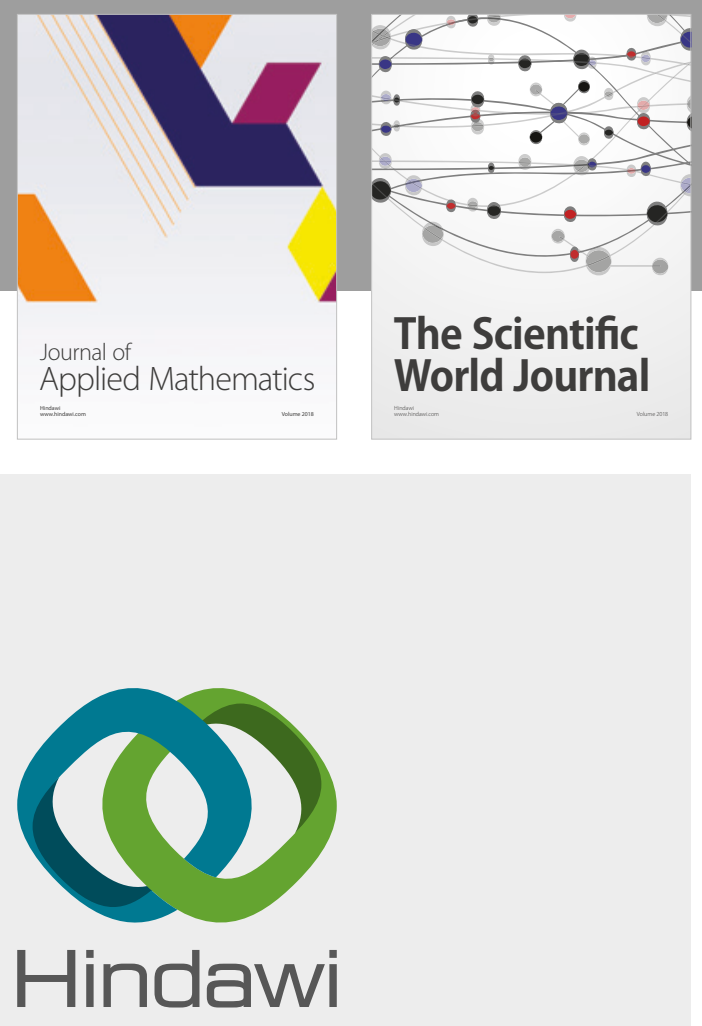

Submit your manuscripts at

www.hindawi.com

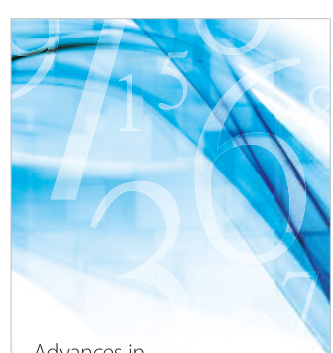

Advances in
Numerical Analysis
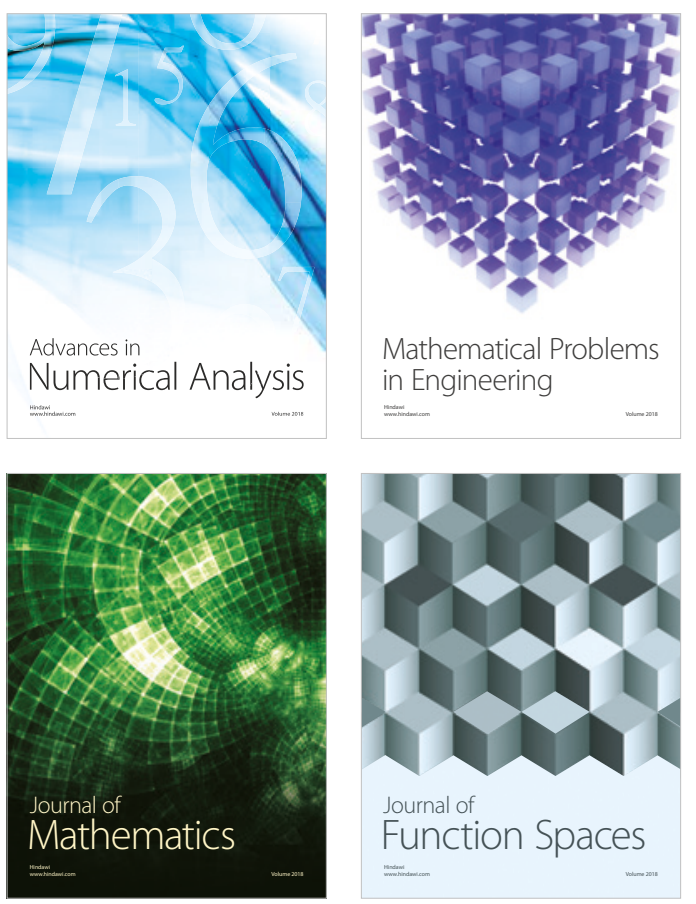

Mathematical Problems in Engineering

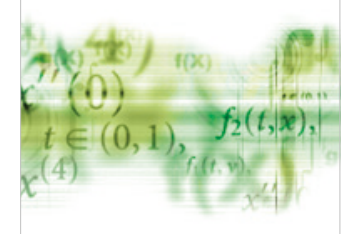

International Journal of

Differential Equations

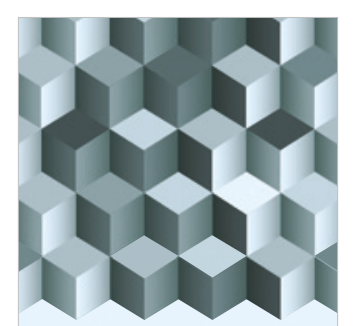

Journal of

Function Spaces
The Scientific

World Journal

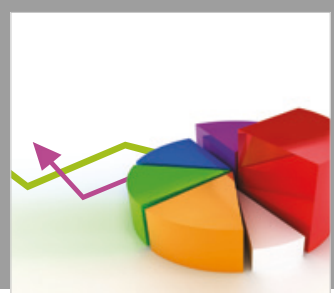

Journal of

Probability and Statistics
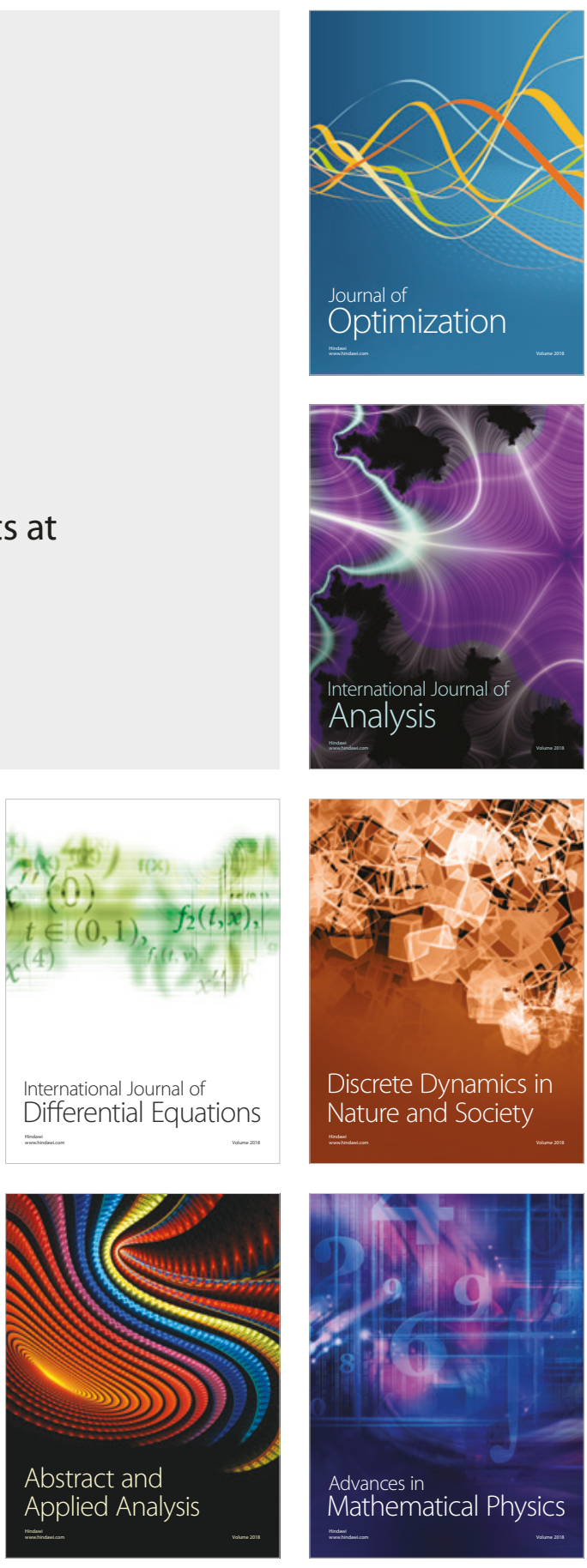